\title{
LA SALUTE MENTALE NELL'ORDINAMENTO COSTITUZIONALE ITALIANO
}

\section{LA SALUD MENTAL EN EL ORDEN CONSTITUCIONAL ITALIANO}

\section{THE MENTAL HEALTH IN THE CONSTITUTIONAL ITALIAN ORDER}

\section{Stefano Rossi ${ }^{2}$}

RESUMEN: El artículo tiene como objetivo examinar el desarrollo de la legislación sobre la salud mental en el contexto del modelo sanitario italiano. La salud mental, en particular la condición jurídica de las presonas que tienen algún transtorno mental, representa un observatorio privilegiado para analizar y destacar, con los materiales que la prespectiva constitucionlista ofrece, las contradicciones que aún persisten en el ordenamiento italiano, así como las dificultades de descartar o afianzar algunas formas de tutela sobre adeterminados derechos y sus líneas evolutivas, en el campo de los derechos civiles y sociales, que han pretendido restituir la "dignidad" social a los llamados "locos". Por tanto, el desarrollo del análisis descrito se propone examinar el carácter irregular y complejo de la salud mental a través de algunos principios que pertenecen al léxico constitucionalista y las incosistencias que han caracterizado al marco jurídico italiano.

Palabras clave: reforma siquiátrica, Ley de Salud Mental, dignidad humana, derechos constitucionales, identidad personal.

RIASSUNTO: L'articolo si propone di esaminare gli sviluppi della legislazione in materia di salute mentale nel contesto del modello sanitario italiano. La salute mentale, e in particolare la condizione giuridica delle persone affette da sofferenza mentale, costituisce un osservatorio privilegiato per analizzare e far emergere, con le risorse che la prospettiva costituzionalistica ci offre, le contraddizioni ancora insite nell'ordinamento, la difficoltà di declinare entro forme di tutela effettiva determinati diritti, ma anche le linee evolutive, nel campo dei diritti civili e sociali, che hanno inteso restituire dignità sociale ai 'folli'. In tali termini, il percorso di analisi che si è inteso intraprendere è volto a vagliare i tratti frastagliati e complessi del tema della salute mentale attraverso alcuni principi propri del lessico costituzionalistico e le ricaduto che hanno caratterizzato il contesto normativo italiano.

Keywords. Psychiatric Reform, Mental Health Act, Dignity, Right, Constitutional Rights, Personal Identity.

SOMmARIO: I. Il diritto alla salute (mentale) nella Costituzione. II. Il laboratorio italiano della salute mentale: la lunga marcia sino alla legge "Basaglia". III. La regionalizzazione della tutela della salute mentale. IV. La sanità nel processo di decentramento e aziendalizzazione. V. I Progetti Obiettivo: direttive per l'attuazione della I. 180/1978. VI. I LEA e la salute mentale: limiti e sfide future. VII.

\footnotetext{
${ }^{1}$ Artículo recibido el 28 de enero de 2015 y aceptado para su publicación el 24 de julio de 2015.

${ }^{2}$ Dottorando di ricerca in diritto pubblico e tributario nella dimensione europea presso il Dipartimento di Giurisprudenza dell'Università degli Studi di Bergamo.
} 
Scienza incerta e Mental Health Policy. VIII. Conclusioni minime: la salute mentale tra legislazione di dettaglio e linee guida.

\section{IL DIRITTO ALLA SALUTE (MENTALE) NELLA COSTITUZIONE}

L'articolo 32 Costituzione, che tutela il diritto alla salute, è indiscutibilmente uno dei più complessi da esaminare, interpretare e applicare, in quanto garantisce un insieme variegato di posizioni giuridiche soggettive. ${ }^{3}$ Tale «fascio di diritti» ${ }^{4}$ esibisce invero aspetti comunemente ritenuti tipici dei diritti sociali (la pretesa che ha ad oggetto una prestazione), quando si presenta come diritto alle cure (gratuite o sotto costo) e aspetti propri dei diritti di libertà (la pretesa che ha ad oggetto un'astensione), quando si presenta come diritto a determinarsi in ordine alle proprie scelte terapeutiche (quindi anche come diritto a non essere curato). ${ }^{5}$ Se ne può cogliere, in tale senso, la natura complessa, che si rappresenta come una costellazione "nella quale assumono rilievo, contemporaneamente ed intrecciate tra loro, pretese a determinati comportamenti pubblici, pretese di astensione, situazioni soggettive di svantaggio". ${ }^{6}$

Complessità che si esprime anche attraverso il paradigma del diritto fondamentale, espressamente previsto dalla lettera della Costituzione solo in relazione alla tutela della salute, e di correlato interesse della collettività a che tutti i componenti godano del miglior stato di salute possibile a mezzo della predisposizione da parte dello Stato delle strutture e dei presidi sanitari più adeguati. ${ }^{7}$ La salute, in questi termini, è un diritto per il singolo e un interesse per la collettività, ed è proprio per ovviare ai rischi insiti in tale asimmetria che ne consegue la sua

${ }^{3}$ La letteratura giuridica su questi temi è molto ampia. Fra i lavori di maggiore interesse, senza pretesa di completezza: Carlassare, L., “L'articolo 32 Costituzione e il suo significato”, in Alessi, R. (a cura di), L'amministrazione sanitaria italiana, Vicenza, Atti del congresso celebrativo del centenario delle leggi amministrative di unificazione, 1967, pp. 105 ss.; Vincenzi Amato, D., Articolo 32, $2^{\circ}$ co., in Comm. Costituzione Branca, Bologna-Roma, Zanichelli-Il Foro Italiano, 1976, pp. 175 ss.; Luciani, M., “Il diritto costituzionale alla salute”, Diritto e società, 1980, pp. 769 ss.; Pezzini, B., “Il diritto alla salute: profili costituzionali", cit., 1983, t. I, pp. 21 ss.; Romboli, R., Articolo 5. Delle persone fisiche. Artt. 1- 10, in Comm. Scialoja-Branca, Bologna-Roma, Zanichelli-Il Foro Italiano, 1988, pp. 225 ss.; Morana, D., La salute nella Costituzione italiana. Profili sistematici, Milano, Giuffrè, 2002.

${ }^{4}$ Principato, L., “Il diritto costituzionale alla salute: molteplici facoltà più o meno disponibili da parte del legislatore o differenti situazioni giuridiche soggettive?", Giurisprudenza costituzionale, 1999, t. II, p. 2513.

${ }^{5}$ Luciani, M., "Sui diritti sociali", in Romboli, R., (a cura di), La tutela dei diritti fondamentali davanti alle Corti costituzionali, Torino, Giappicchelli, 1994, p. 96.

${ }^{6}$ Pezzini, B., op. cit., p. 25.

${ }^{7}$ Sotto tale aspetto la "pretesa di astensione e pretesa di prestazione si intrecciano e si fondono, presentandosi come le due facce di una moneta; la libertà individuale si pone in definitiva come il presupposto, la radice, di un diritto strutturato in forma articolata in varie direzioni, sia positive che negative, che corrispondono ai diversi profili che la norma prende in considerazione nell'attribuire una tutela". Ibidem, p. 72. 
tutela in via primaria ed immediata come modo di essere della persona ed espressione della sua sfera di libertà, ${ }^{8}$ che non può quindi subire alcuna deminutio nel rapporto-confronto con le esigenze collettive.

Sicchè, in una prospettiva dinamica, il diritto alla salute —che, in quanto diritto fondamentale, si collega da un lato con la tutela generale della personalità (articulo 20. Costituzione), con i diritti fondamentali di eguaglianza, dignità (articolo 3o. Costituzione) e libertà della persona (articolo 13 Costituzione), dall'altro con le norme che garantiscono le concrete estrinsecazioni sociali della persona- esprime in via prevalente la somma degli ottativi del soggetto ovvero delle pretese non aprioristicamente determinabili la cui soddisfazione è presupposto fattuale del miglioramento degli standards qualitativi dell'esistenza, essenziali per il libero svolgimento della

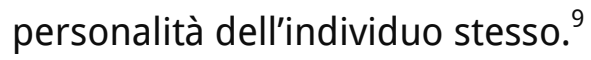

In tale ottica si può scorgere un percorso parallelo tra lo sviluppo della nozione di salute che, transitando da una dimensione monadica ad una relativizzata, ha finito con il comprendere non soltanto funzioni biologiche in senso stretto, ma anche capacità logiche, affettive e relazionali ${ }^{10} \mathrm{e}$ la correlata espansione del campo di tutela affidato all'articolo 32 Costituzione, disposizione che dalla semplice tutela della 'salute pubblica' si è resa 'trasformatore permanente' delle istanze individuali e sociali (dalla tutela della salute dei lavoratori alla salubrità dell'ambiente, dal diritto al risarcimento del danno biologico sino all'affermazione del consenso informato) in diritti giustiziabili.

A fronte del profilo attinente ai diritti individuali connessi alla salute, non meno rilevante appare il suo riflesso quale interesse collettivo, che è rivolto all'acquisizione di uno stato di

\footnotetext{
${ }^{8}$ Secondo Onida, V., "Costituzione italiana”, in Digesto discipline pubblicistiche, Agg. VI, Torino, Utet, 1989, pp. 29-330.

"...la Costituzione si ispira anzitutto all'idea-forza della centralità e del primato della persona umana, considerata come soggetto di diritti in certo senso anteriori a qualsiasi riconoscimento da parte dello Stato, e quindi non condizionati a finalità collettive di qualsiasi genere, anche se limitati e limitabili — ma non in qualsiasi direzione e misura - in vista di esigenze degli altri individui della comunità, e quindi in vista dell'adempimento dei doveri inderogabili di solidarietà". Luciani, Anche M., "Sui diritti sociali", cit., p. 772.

${ }^{9}$ Cocconi, M., Il diritto alla tutela della salute, Padova, Cedam, 1998, p. 65.

${ }^{10}$ D'Arrigo, C., "Salute (diritto alla)", in Enciclopedia del diritto, Agg. IV, Milano, Giuffrè, 2001, p. 1013. Così da una definizione attenta puramente al dato biologico, dell'assenza di malattia, si è passati a un concetto assai più ampio, che comprende l'aspetto dinamico del miglioramento del benessere e guarda alla complessità dell'equilibrio tra soma e psiche. Specularmente, anche il concetto di malattia si presta a una formulazione estensiva, che dall'originario riferimento all'alterazione anatomo-funzionale si proietta verso l'area più indefinita delle sociopatie, consistenti nei disagi e nei disturbi variamente connessi con il contesto socio-culturale e ambientale.
} 
benessere generale riguardante il complesso della popolazione ${ }^{11}$, al di là quindi della condizione individuale di ciascuno. Tuttavia è bene sottolineare il duplice aspetto entro cui si incardina l'interesse collettivo, inteso sia come insieme di prestazioni sanitarie (anche preventive) atte a garantire la salute pubblica e destinate alla generalità della popolazione sia come "dovere dell'individuo di non ledere né mettere in pericolo con il proprio comportamento la salute altrui, secondo il principio per cui il proprio diritto trova limite nel reciproco riconoscimento e nell'eguale protezione del diritto degli altri" (Corte Costituzione núm. 258/1994).

È bene sottolineare come non sia ammissibile far discendere dal profilo collettivo della salute un dovere generale, soggetto a sanzione, di mantenersi in buona condizione psicofisica, in quanto "la preminenza nella tutela della salute dell'aspetto soggettivo personale rispetto a quello collettivo rende inaccettabile un sacrificio della libera determinazione individuale se non in presenza di rischi per lo stato di salute altrui". ${ }^{12}$ L'interesse collettivo, infatti, rappresenta un limite esterno, e non interno, al diritto individuale, ovvero una barriera che ne circoscrive l'ampiezza senza tuttavia condizionarne il godimento. Peraltro, secondo la lettura ormai consolidata della dottrina ${ }^{13}$ e della giurisprudenza costituzionale, ${ }^{14}$ l'imposizione di un determinato trattamento sanitario, stabilito per legge, risulta legittima solo quando sia in gioco non soltanto la salute del singolo in quanto tale, ma anche, e direttamente, l'interesse collettivo alla salute. Ciò si evince dalla lettura combinata dei due commi dell'articolo 32 Costituzione, per cui il carattere di limite esterno alla libertà individuale dell'interesse della collettività impedisce qualsiasi condizionamento intrinseco che ne trasformi in senso funzionale la natura: ${ }^{15}$ la condizione richiesta per imporre un trattamento sanitario obbligatorio, ovvero la coesistenza della finalità di tutela della salute individuale e di quella collettiva, si configura quindi come un'endiadi, volta essenzialmente a proteggere i valori che integrano il profilo assiologico della persona umana.

\footnotetext{
${ }^{11}$ La tutela della salute ha anche implicazioni collettive, nascenti dall'esigenza di combattere forme di morbilità epidemiche e di garantire, in generale, la salubrità, anche attraverso la limitazione della libertà dei singoli.

${ }^{12}$ Cocconi, M., op. cit., p. 93. Il trattamento sanitario deve comunque servire alla protezione dell'interesse della collettività alla salute generale e non può essere finalizzato al perseguimento di qualunque interesse pubblico.

${ }^{13}$ Carlassare, L., op. cit., pp. 110 y ss.; Vincenzi Amato, D., op. cit., pp. 174 y ss.; Pezzini, B., op. cit., pp. 30, ss., 63 y 64; Luciani, M., Salute I) diritto alla salute-dir. Costituzione, cit., pp. 9 y 10.

${ }^{14}$ In particolare Corte Costituzione, 22 giugno 1990, núm. 307, su cui Giardina, F., “Vaccinazione obbligatoria, danno alla salute e responsabilità dello Stato", Giurisprudenza costituzionale, 1990, pp. 1880 ss.; Modugno, F., “Chiosa a chiusa. Un modello di bilanciamento di valori", Giurisprudenza italiana, 1995, t. 1, pp. 64 y ss.

${ }^{15}$ Morana, D., op. cit., pp. 169, 172 y ss. Sulla disciplina dei trattamenti sanitari obbligatori.
} 
Se dunque si scrutina la sostenibilità della disciplina dei Tso in materia di salute mentale alla luce dei parametri costituzionali ne emerge un quadro critico, nella misura in cui appare sfuggente quell'interesse alla tutela della salute collettiva tale da giustificare l'imposizione di un trattamento ad un soggetto con sofferenza psichica, ${ }^{16}$ non rinvenibile certo - in forza di un meccanismo surrogatorio - nel vetusto criterio della pericolosità sociale. ${ }^{17}$

Il tema della sicurezza rappresenta, certo, una delle questioni che balzano maggiormente all'ordine del giorno e la psichiatria è spesso chiamata in causa da una società che, impaurita, chiede protezione contro una violenza vista come incomprensibile, imprevedibile, insensata e frutto di un black-out della ragione che è possibile aspettarsi nel malato di mente.

Tali esigenze collettive non possono tuttavia trovare risposta in un esasperato ricorso ai trattamenti coattivi o in forme di segregazione neo-manicomiale, ${ }^{18}$ ma debbono essere affrontate nel quadro di un'assistenza di prevenzione e cura a carattere territoriale, di prossimità, che sappia coinvolgere il paziente, la sua famiglia e il contesto comunitario in cui questi vive. ${ }^{19}$

L'interesse collettivo alla salute mentale si invera dunque in efficaci programmi e servizi di prevenzione, diagnosi e cura, in una psichiatria migliore nella sua capacità di incontrare l'altro che necessita di aiuto e anche di erogare trattamenti caratterizzati da un più alto grado di

\footnotetext{
${ }^{16}$ Vincenzi Amato, D., op. cit., p. 192. secondo cui «questo legame tra la salute del singolo e salute della collettività sussiste probabilmente solo con riguardo alle malattie contagiose, ed è senz'altro da escludere in presenza di malattie mentali che soltanto in modo indiretto - proprio attraverso, cioè, la eventuale pericolosità del soggetto - possono minacciare la salute degli altri"; Vecchietti, A. M., op. cit., pp. 105-107 secondo cui "resta, pertanto, il dubbio che ciò che deve avere preoccupato maggiormente il legislatore sia stato, subito e con validità di effetti, attuare intanto le forme di coercitive della libertà solo a «questi» cittadini, certamente ancora molto scomodi, con l'apparente intenzione di tutelare la loro salute, ma con il recondito fine sia di proteggere da essi la società, sia di modificarne il comportamento, attraverso sistemi autoritari di controllo delle devianze".

${ }^{17} \mathrm{Nel}$ contesto di una visione dinamica e relazionale della salute, si potrebbe individuare il contenuto dell'interesse collettivo nell'interesse della comunità alla conservazione delle capacità e funzionamenti (per usare il linguaggio della Nussbaum) del singolo a realizzare sé stesso anche nel contesto delle formazioni sociali in cui è inserito.

${ }^{18}$ Preoccupanti, in particolare, risultano i progetti di legge (ripresentati ad ogni legislatura) volti ad estendere misure di riduzione della libertà personale, travestite da trattamento terapeutico, che intendono dilatare i tempi di ricovero coatto in strutture residenziali trasformate così in piccoli manicomi, dove ancora si chiede allo psichiatra di essere un severo custode di spazi chiusi, nei quali la dimensione terapeutica rischia di perdere valori e significati.

${ }^{19}$ Bisogna tenere pur conto, in questi casi, che la richiesta rivolta alla psichiatria e alle sue istituzioni rischia di non essere una richiesta di "cura", ma d'altro genere: sono il contenimento e il controllo (anche chimico) dei comportamenti, I'"esilio" a una certa distanza (meglio se lunga) e per qualche tempo (il più possibile) dal contesto sociale e dalla famiglia, e, al più, un intervento esclusivamente pedagogico-educativo che con la cura psichiatrica non dovrebbe essere confuso. Ferrannini, I. y Peloso, P. F., “I trattamenti senza consenso in psichiatria e in medicina tra norme, culture e pratiche. Appunti per una discussione", Noos, 2012, t. 1, pp. 40 y ss.
} 
appropriatezza, insieme ad un contesto sociale più aperto verso la possibilità di integrazione delle persone cui capita di fare nella propria vita esperienza della malattia mentale e più attento a non colpirle con atteggiamenti di stigmatizzazione. ${ }^{20}$

È nel solco di tale narrazione che va inserito il racconto della salute mentale che, pur non essendo più isolabile dalla salute in sé intesa, ${ }^{21}$ ha vissuto e vive un travaglio concettuale, ${ }^{22}$ che si riflette inevitabilmente sui profili giuridici del suo disciplinamento.

Sembra infatti impossibile risolvere la "crisi" della psichiatria attraverso l'individuazione di un concetto di malattia mentale scientificamente valido, o, quantomeno, scientificamente 'più valido' degli altri, laddove nemmeno il tentativo di razionalizzazione operato attraverso il sistema DSM ${ }^{23}$ è stato in grado di offrire una risposta rassicurante. Dato che la costruzione giuridica della scienza non può più limitarsi a recepire le proposizioni che quest'ultima propugna, è necessario passare a una condizione di reciproco scambio critico in cui il diritto, trovandosi a regolare una scienza incerta, deve, da un lato, esprimere valutazioni sul sapere scientifico, e, dall'altro, rendere ragione (anche) della scientificità delle proprie scelte, sciogliendo prescrittivamente un 'nodo' non districato del sapere descrittivo. ${ }^{24}$

Tuttavia, al di là di tale aspetto che pur risulta centrale nella definizione del rapporto tra diritto e scienza, ${ }^{25}$ un dato appare, specie in questo campo, indiscutibile, ovvero che il mutamento di

\footnotetext{
${ }^{20}$ Albanesi, C. y Migani, C., Il lavoro di rete nella promozione della salute mentale: teorie e pratiche per un modello di intervento, Roma, Carocci, 2004, pp. 36 y ss.
}

${ }^{21}$ In tal senso "Non c'è salute senza salute mentale" come sancito nella Conferenza interministeriale europea per la Salute mentale svoltasi a Helsinki nel 2005; principio poi ribadito nel Libro verde per la salute mentale approvato dal Parlamento europeo nel 2007.

${ }^{22}$ In questo senso con l'editoriale intitolato "Psichiatria: il tramonto delle certezze", Pier Francesco Galli e Paolo Migone aprono il núm. 1/2013 della rivista Psicoterapia e Scienze umane; con grande autorevolezza, nello stesso senso, si veda Angell, M., “L'epidemia di malattie mentali e le illusioni della psichiatria”, cit., 2012, t. 2, pp. 263-282.

${ }^{23}$ Il manuale internazionale di psicodiagnostica più diffuso e accreditato "dell'American Psychiatric Association", L'ultima versione è il DSM-V-TR, AA. VV., Diagnostic and Statistical Manual of Mental Disorders: Dsm-5, American Psychiatric Publishing, 2013. L'altra classificazione dei disturbi mentali è quella dell'Organizzazione Mondiale della Sanità, I'ICD-10 (International Classification of Diseases) approvata nel 1990 durante la 43 Assemblea mondiale della Sanità dell'OMS e utilizzata a partire dal 1994.

${ }^{24}$ Tallacchini, M., “Biotecnologie e diritto della scienza incerta”, Notizie di Politeia, 1999, 54, pp. 98-100.

${ }^{25}$ Vaccari, V. G., “I Fatti scientifici nella Giurisprudenza Costituzionale. Il caso del diritto alla salute”, Tesi di dottorato di ricerca in Diritto costituzionale, Ciclo XXI, Università di Ferrara, pp. 110 ss.; D’Amico, G., "Scienza e diritto nella prospettiva del giudice delle leggi", SGB ed., Messina, 2008; ID., "I dubbi della scienza al vaglio della Corte costituzionale: dalle incertezze della scelta alle incertezze del diritto (materiali giurisprudenziali)", in D'Aloia, A. (a cura di), Biotecnologie e valori costituzionali. Il contributo della giustizia costituzionale, Torino, Giappichelli, 2005, pp. 237 ss.; 
approccio alla malattia di mente - dall'impianto custodialistico e repressivo alla convinzione che la socializzazione e partecipazione del malato possieda essa stessa un'efficacia terapeutica - ha determinato, dapprima, un'evoluzione della disciplina pubblicistica relativa ai trattamenti sanitari nel segno della volontarietà e, successivamente, una riforma dello statuto privatistico degli infermi di mente e della loro tutela, volta a restituire soggettività alle persone vulnerabili.

Inquadrare la salute mentale nella costellazione del diritto alla salute consente di mettere in piedi un dispositivo di linguaggio, un codice, un'utopia in grado di occuparsi della sofferenza psichica che —come ha scritto Paolo Zatti— significa saper come:

...regolare rapporti e decisioni che si inseriscono nelle condizioni di fragilità, di fatica, di malattia e di battaglia, di handicap, di non autosufficienza, di declino delle forze e della vitalità, di vecchiaia, di attesa e di desiderio di morire: le condizioni che tutti gli esseri umani incontrano in qualche punto tra la nascita e la morte, e che li conducono in quell'esperienza in cui, come dice Levine, «la mente si consuma e il cuore diviene di brace». ${ }^{26}$

Sicchè lo sguardo del giurista, in tale prospettiva, incontra il malato, non la malattia; nella sua mente non cerca di decifrare i segni di una patologia (per valutarne l'incapacità o l'imputabilità), ma vuole conoscerne la biografia, il che impedisce alla soggettività del paziente (fatta di rapporti, diritti e modi di vita) di scomparire dietro l'oggettività di segni sintomatici e, al contempo, di ridurne la complessità entro quella forzata grammatica nosografica attraverso cui si classifica la malattia per classificare anche la persona.

\section{IL LABORATORIO ITALIANO DELLA SALUTE MENTALE: LA LUNGA MARCIA SINO ALLA LEGGE "BASAGLIA"}

La legge 180/1978 è il frutto di una rivoluzione "copernicana", che, nel porre la persona -e non più la malattia - al centro dell'universo della sofferenza psichica, ne ha fatto il luogo di riflessione della psichiatria, di attenzione ed azione dei servizi e infine di discussione nel contesto sociale.

La condizione del malato di mente è divenuta, in tal senso, paradigma per una riflessione complessiva sui meccanismi e le forme di disciplinamento della società, ovvero sul ruolo dell'istituzione quale insieme di apparati scientifici, legislativi, amministrativi, di codici di riferimento culturale e di rapporti di potere strutturati attorno al binomio malattia-pericolosità.

\footnotetext{
Id., "La Corte e lo stato dell'arte (prime note sul rilievo del progresso scientifico e tecnologico nella giurisprudenza costituzionale)", in Malfatti, E., Romboli, R., y Rossi, E. (a cura di), Il giudizio sulle leggi e la sua diffusione, Torino, Giappichelli, 2002, pp. 431 y ss.

${ }^{26}$ Zatti, P., “Oltre la capacità”, in Ferrando, G. y Visintini, G. (a cura di), Follia e diritto, Torino, Bollati Boringhieri, 2003, p. 54.
} 
Al fine di comprendere la complessità del cammino percorso per superare consolidati stereotipi e crittotipi ${ }^{27}$ che connotavano la legislazione psichiatrica italiana, basti pensare che la legge 14 febbraio 1904, núm. 36 sui "Manicomi e sugli alienati” —entrata a regime nel 1909 con l'approvazione del relativo regolamento di attuazione núm. 615- era strutturata intorno alla centralità dell'ordine pubblico, ponendo in primo piano il bisogno di tutelare, di salvaguardare la "società dei sani" dai malati di mente, subordinando quindi la cura alla custodia, nell'ottica secondo cui la malattia mentale, concepita in termini strettamente biologici, era da considerarsi come dato naturale, incurabile e irreversibile. ${ }^{28}$

L'orientamento della legislazione giolittiana — volto a realizzare forme di controllo sociale e al contempo di indirizzo del costume- si desumeva peraltro dalle stesse condizioni atte a legittimare l'internamento in manicomio, disposto ordinariamente dal procuratore e in caso di urgenza dall'autorità di pubblica sicurezza, ovvero la pericolosità per sé o per altri o il pubblico scandalo ingenerato da soggetti che non potessero essere custoditi e curati convenientemente al di fuori del manicomio. ${ }^{29} \mathrm{~A}$ completare il quadro vi era il meccanismo che prescriveva, a seguito del provvedimento di internamento definitivo, una sorta di "cancellazione civile" che privava il malato non solo dei diritti politici, ma anche della capacità di agire attraverso interdizione e nomina di un tutore per l'amministrazione dei beni patrimoniali.

\section{$\underline{27}$}

Sacco, R., Introduzione al diritto comparato, Torino, Giappichelli, 1980, pp. 3 y 109. Definisce il concetto di crittotipo come un "modello (in particolare regola) non verbalizzato cioè rimasto inespresso nell'ordinamento considerato".

${ }^{28}$ Merlini, S., "Libertà personale e tutela della salute mentale: profili costituzionali", Democrazia e diritto, 1970, t. 1-2, pp. 61 ss.; BruscugliA, L., Infermità di mente e capacità di agire. Note critiche e sistematiche in relazione alla legge 18 marzo 1968, Milano, Giuffrè, 1971, núm. 431, pp. 8 y ss.; Bruscuglia, L., Busnelli, F. D. y Galoppini, A., "Salute mentale dell'individuo e tutela giuridica della personalità", Rivista trimestrale di diritto e procedura civile, 1973, pp. 661-663; Vecchietti, A. M., op. cit., pp. 2 y ss.; Morello, M., I malati di mente dalla legislazione preunitaria alla legge Basaglia, San Marino, Guardigli, 2008, pp. 99 y ss.; in controtendenza Visintini, G., "La riforma in tema d'assistenza psichiatrica. Problematica giuridica", Politica del diritto, 1982, t. 3, pp. 447 ss. Secondo cui "le legge, di per sé, rappresentava un progresso rispetto al passato: essa individuava, per la prima volta, come pubblico, cioè da gestire dallo Stato, l'interesse alla sanità mentale... tutti i motivi di critica erano tuttavia da imputare più che alla legge, alla sua interpretazione ed applicazione".

${ }^{29}$ La malattia di mente, più che riguardare la materia della sanità, apparteneva a quella della pubblica sicurezza, tanto da rientrare anche nel testo unico di pubblica sicurezza, il quale prevedeva a carico degli esercenti la professione sanitaria l'obbligo di denunciare entro due giorni all'autorità di pubblica sicurezza tutte "le persone da loro assistite o esaminate affette da malattia mentale o da grave infermità psichica, le quali dimostrino o diano sospetto di essere pericolose per sé e agli altri" (articolo 153 r.d. 18 giugno 1931, núm. 773); correlativamente, seppur a titolo contravvenzionale, il codice penale sanzionava una serie di condotte (artt. $714,715,716,717$ c.p.) che riflettevano la sussistenza di obblighi di custodia o di denuncia. 
Neanche l'avvento della Costituzione contribuì ad un rapido adeguamento dell'arcaico tessuto normativo ai principi di uguaglianza e tutela delle diritti individuali e sociali, laddove ad una psichiatria internazionale che, negli anni '50, si apriva ai contributi della psicoanalisi, della fenomenologia e della sociologia, faceva riscontro, in Italia, una classe medica in larga parte arroccata su posizioni organiciste, sull'assolutizzazione della diagnosi e sul legame -esplicito nella legge del 1904 - tra esigenze di ordine pubblico e internamento manicomiale.

Solo a partire dagli anni '60, il periodo del cd. "disgelo costituzionale", ${ }^{30}$ un insieme (irripetibile) di condizioni politiche e sociali ${ }^{31}$ rese possibile l'avvio di un percorso di riforme volte a superare i residui e le distorsioni del regime fascista, dando piena attuazione alla Carta costituzionale attraverso l'apertura di nuovi spazi di libertà. In questo contesto si colloca la legge 18 marzo 1968, núm. 431 che rivestì un ruolo centrale nella modernizzazione della legislazione psichiatrica. La nuova normativa, pur parziale nei suoi contenuti, veniva ad incidere su meccanismi che contribuivano al fenomeno di stigmatizzazione ed istituzionalizzazione del malato di mente. Con essa infatti veniva abrogato l'obbligo di annotazione del ricovero in manicomio nel casellario giudiziario, si prevedeva inoltre la possibilità di ricovero volontario in ospedale psichiatrico ed inoltre di trasformazione dei ricoveri coatti in volontari, spezzando per la prima volta il legame tra malattia mentale e pericolosità. Sul piano organizzativo si stabiliva che ciascun ospedale psichiatrico dovesse essere costituito "da due a cinque divisioni, ciascuna delle quali con non più di 125 posti letto", stanziando risorse per l'adeguamento delle strutture e delle piante organiche di ciascun istituto. La legge 'Mariotti', ispirata ai principi della psichiatria territoriale di settore, prevedeva anche la creazione dei Centri di igiene mentale (Cim) in grado di fungere da servizi di medicina preventiva di prossimità.

La breccia aperta nell' 'istituzione totale' del manicomio venne ampliata da una prassi coraggiosa e prospettica, portata innanzi da psichiatri e amministrazioni locali illuminate, che valorizzò l'attività di prevenzione, diagnosi e presa in carico del malato attraverso servizi di tipo ambulatoriale, affiancati dalla creazione di laboratori protetti, centri diurni e case-famiglia,

\footnotetext{
${ }^{30}$ Pizzorusso, A., “Il disgelo costituzionale”, in Storia dell'Italia repubblicana, Torino, Einaudi, 1995, vol. II., t. 2, pp. 136 y ss.

${ }^{31}$ Ginsborg, P., Storia d'Italia dal dopoguerra ad oggi, Torino, Einaudi, 2006, pp. 404 y ss.; Revelli, M., “Movimenti sociali e spazio pubblico", in Storia dell'Italia repubblicana, La trasformazione dell'Italia. Sviluppo e squilibri, Torino, Einaudi, vol. II, t. 2, 1995; sotto il profilo della storia della psichiatrica Cassata, F. y Moraglio, M. (a cura di), Manicomo, società e politica. Storia, memoria e cultura della devianza mentale dal Piemonte all'Italia, Pisa, Bfs, 2005; Id. (a cura di), Ci sarà una festa. Per una storia sociale della psichiatria, in "L'indice dei libri del mese", 6, 2003.
} 
collocati prevalentemente in zone non marginali del tessuto urbano e sociale e che in molti casi erano aperti a tutta la popolazione. ${ }^{32}$

La legge 431/1968 consentì agli operatori di uscire nel territorio e, al contempo, di sperimentare pratiche volte a forzare la realtà manicomiale, mirando esplicitamente al suo smantellamento. Dentro quelle esperienze di de-istituzionalizzazione la nuova psichiatria riuscì a costruire anche rilevanti rapporti con il territorio e sperimentare nuove forme terapeutiche nella relazione diretta con i pazienti, abbattendo le barriere gerarchiche e mettendo in discussione $\mathrm{i}$ saperi professionali di cui erano portatori i diversi tipi di operatori (medici, infermieri, assistenti ospedalieri).

Sicche si può notare come la svolta, concretatasi nella legge 13 maggio 1978, núm. 180, fosse, nel contesto sociale e nelle pratiche terapeutiche, ormai maturata da diversi anni attraverso quel sapere esperienziale accumulato a partire dagli anni '60; a rendere però ineludibile la scelta fu l'approssimarsi di un referendum, proposto dai radicali, volto all'abrogazione della disciplina del 1904, il che costrinse le forze parlamentari, ad ampia maggioranza, ad approvare una legge stralcio, disciplinante il solo settore della salute mentale, con l'espressa intenzione di riassorbirne i contenuti nella più complessiva riforma istitutiva del Servizio Sanitario Nazionale (I. 23 dicembre 1978, núm. 833).

Nel regolare il trattamento della malattia mentale, il legislatore -abbandonata completamente l'ottica asilare e para-detentiva caratterizzante la vecchia legge del 1904definisce una scala di valori che vede il primato della salute dell'uomo, della sua libertà e dignità su ogni altro interesse (v., in particolare, artt. 10., $2^{\circ}$ co., e $20 ., 2^{\circ}$ co., lett. g), I. 833/78, e articolo 10., $2^{\circ}$ co., I. 180/78). Si postula un diverso approccio alla salute mentale, modificando gli obiettivi fondamentali dell'intervento pubblico — dal controllo sociale dei malati alla promozione della salute e prevenzione dei disturbi mentali- spostando di conseguenza l'asse portante dell'intervento istituzionale dal ricovero ospedaliero alla definizione di servizi territoriali.

Sul piano organizzativo, ciò si traduce nell'inserimento organico dei servizi psichiatrici nell'ambito dei servizi generali, in modo da eliminare - pur nella specificità delle misure terapeutiche- ogni forma di discriminazione e di segregazione verso i settori di cura delle infermità mentali, e al fine di favorire, al contempo, il recupero e il reinserimento sociale delle persone con disturbo psichico (articolo 20., $2^{\circ}$ co., lett. g), I. 180/1978)

\footnotetext{
${ }^{32}$ Sulle esperienze di quegli anni Basaglia, F. (a cura di), L'istituzione negata, Torino, Einaudi, 1968; AA. VV., La pratica della follia. Atti del 10. Convegno nazionale di Psichiatria democratica Gorizia, 22-23 giugno 1974, Centro Internazionale di Studi e Ricerche, Venezia, 1975; Basaglia, F. y Tranchina, P. (a cura di), Autobiografia di un movimento: 1961-1979: dal manicomio alla Riforma sanitaria, Firenze, Upi, 1979; Venturini, E., Il giardino dei gelsi. Dieci anni di antipsichiatria italiana, Torino, Einaudi, 1979.
} 
In termini direttivi, inoltre il trattamento dell'infermo in condizioni di degenza ospedaliera risulta, per esplicita scelta del legislatore, ridotto ad una forma di intervento assolutamente residuale e transitoria, ${ }^{33}$ venendo indicati quali luoghi in cui attuare, di norma, gli interventi terapeutici e riabilitativi, i Centri e i presidii psichiatrici extra-ospedalieri; mentre per il ricovero ospedaliero si prevede la creazione di specifici reparti di diagnosi e cura all'interno delle strutture dipartimentali per la salute mentale (articolo $34,5^{\circ}$ co., I. 833/78 e articolo 6o., $2^{\circ}$ e $3^{\circ}$ co., I. 180/78).

Quanto agli ospedali psichiatrici, la stessa legge -oltre a disporre il divieto di nuove costruzioni- prevede il graduale superamento di quelli esistenti (articolo $64,1^{\circ}$ e $3^{\circ} \mathrm{co} .1 .833 / 78$ e articolo 7o., $5^{\circ}$ e $6^{\circ}$ co. I. 180/78), condizione realizzatasi solo nel 1998.

Con riguardo agli accertamenti e ai trattamenti sanitari, la cui disposizione è condizionata al generale principio di volontarietà, si stabilisce, in via d'eccezione, una disciplina snella che, nell' ‘amministrativizzare' il procedimento di imposizione del trattamento obbligatorio (Aso e Tso), ${ }^{34}$ ne circoscrive la responsabilità dell'adozione entro il circuito formato da sapere medico e autorità locale delegata alla tutela della salute pubblica, con una funzione esterna e di garanzia attribuita al giudice tutelare.

Il legislatore del 1978 ha preferito erigere l'impianto del dispositivo trattamentale sul binomio necessità/rifiuto delle cure, che ne rappresenta il corrispettivo concreto e molto più obiettivabile.

Esso rappresenta, infatti, l'asse portante della condizione in cui un rifiuto (in varie forme) al trattamento non può essere accettato in quanto potenzialmente lesivo per il diritto alla salute del paziente e al contempo per il correlato interesse della collettività: su questo paradigma si delineano i modelli di presa in carico e di prevenzione del drop-out così come il tentativo di costruzione della relazione terapeutica, nella misura in cui si prevede che i trattamenti debbano

\footnotetext{
${ }^{33}$ La degenza ospedaliera non solo si caratterizzava come strumento da attuarsi in casi eccezionali e straordinari, ma soprattutto veniva ad assolvere una funzione esclusivamente terapeutica e non più, come in passato, di difesa sociale. Ciò era il portato della nuova visione della malattia mentale, considerata come una forma di malattia al pari di altre, il cui approccio mira non più alla ghettizzazione ma, al contrario, al pieno recupero alla società del malato sotto ogni profilo.

${ }^{34}$ Anche con riguardo al trattamento sanitario obbligatorio, permane la regola generale del trattamento extraospedaliero. La legge, difatti, ipotizza i casi in cui il trattamento obbligatorio della malattia mentale può prevedere che le cure vengano prestate in situazione di degenza ospedaliera: per darvi luogo, oltre alla presenza di alterazioni psichiche tali da richiedere urgenti interventi terapeutici, e al fatto che gli stessi non vengano accettati dall'infermo, si precisa che devono sussistere condizioni e circostanze che non permettano di adottare tempestive ed idonee misure extra-ospedaliere (articolo 34 I. 833/78; articolo 20., $2^{\circ}$ co. I. 180/78).
} 
“essere accompagnati da iniziative rivolte ad assicurare il consenso e la partecipazione da parte di chi vi è obbligato" (articolo 33, I. 833/1978). ${ }^{35}$

Se l'obiettivo essenziale perseguito attraverso la riforma è stato rappresentato indiscutibilmente dalla chiusura dei manicomi nel segno dei diritti, sostituendo all' Asylum un sistema di cura senza custodia, l'impatto, a livello normativo, sociale e culturale, del passaggio da una cura-controllo del disturbo psichiatrico, attuata di norma in condizioni di degenza ospedaliera, ad una gestione della medesima con netta predominanza del momento territoriale ambulatoriale e domiciliare, si è concretato in uno work in progress in costante evoluzione. E non poteva essere altrimenti in un contesto connotato da bisogni complessi e composti, in cui l'approccio terapeutico non si può limitare all'aspetto sanitario o farmacologico ma deve coinvolgere anche il vissuto esistenziale del malato e della sua famiglia: sicchè i servizi devono farsi flessibili e 'adattivi' misurandosi, se necessario, con estenuanti itinerari di rassicurazione, di sostegno e di spiegazioni nei confronti degli utenti e dei loro congiunti, in un continuo lavoro di proposizione di quanto può effettivamente essere fatto a beneficio del malato, ponendo attenzione alla sua essenza di persona umana sofferente, piuttosto che all'astratto timore della distruttività e pericolosità del disturbo mentale da cui è afflitto.

\section{LA REGIONALIZZAZIONE DELLA TUTELA DELLA SALUTE MENTALE}

Come si è visto l'assetto del Sistema sanitario italiano si presenta allora tutt'altro che statico, influenzato com'è dal sostrato di una posizione giuridica soggettiva in continua evoluzione quale il diritto alla salute. Di questa dinamicità è espressione l'evoluzione stessa della legislazione sanitaria, prima e dopo il 1978.

In questo quadro, il fenomeno di regionalizzazione dell'assistenza sanitaria in ambito psichiatrico ha vissuto due stagioni: una prima, post I. 180/1978, durante la quale l'attuazione della riforma si è prolungata indeterminatamente nel tempo, cristallizzando "situazioni di passaggio" che avrebbero dovuto essere superate in tempi brevi ed una seconda fase, quella dei Progetti Obiettivo, ${ }^{36}$ che hanno rappresentato una svolta nella piena ed effettiva attuazione della lettera e dello spirito della riforma.

È bene rammentare che sino al 1968 l'assistenza sanitaria era gestita per il profilo ospedaliero da organismi autonomi, pubblici e privati, spesso con funzioni miste anche di beneficenza e istruzione, sulla base del dettato della legge Crispi del 1890, mentre l'assistenza medico-generica,

\footnotetext{
${ }^{35}$ Sulla disciplina dei trattamenti sanitari obbligatori si veda Vecchietti, A. M., op. cit., pp. 50 ss.; per alcuni profili critici Rossi, S., "Salute mentale e dignità della persona. Profili per un dialogo costituzionale", Atti del 2o. Seminario annuale del 'Gruppo di Pisa' dei dottorandi delle discipline giuspubblicistiche dedicato allo "Lo studio delle fonti del diritto e dei diritti fondamentali in alcune ricerche dottorali", 20 de septiembre de 2013, Roma, in www.gruppodipisa.it.

${ }^{36}$ Per la cui trattazione si rinvia all'apposito paragrafo del testo, considerandone quindi gli effetti sul contesto regionale.
} 
specialistica e farmaceutica veniva assicurata tramite l'organizzazione mutualistica. Entro questo quadro la gestione dei manicomi, dei relativi servizi e del personale erano di esclusiva competenza delle amministrazioni provinciali, che hanno continuato a svolgere tale funzione anche dopo l'approvazione della riforma nella lunga fase di transizione che ne è seguita.

Un primo passo verso la regionalizzazione dell'organizzazione della tutela della salute mentale venne compiuto con la legge 22 luglio 1975, núm. 382, con la quale si fissarono i principi e criteri in base ai quali il governo, entro 12 mesi, avrebbe dovuto emanare decreti diretti a completare il trasferimento delle funzioni amministrative inerenti le materie di cui all'articolo 117 Costituzione, nonché degli uffici e del personale alle regioni. In particolare con il d.P.R. 24 luglio 1977, n. 616 furono affidate alle regioni le funzioni di programmazione e di organizzazione dei servizi, nonché le tipologie e modalità delle prestazioni inerenti alla materia dell' "assistenza sanitaria ed ospedaliera". ${ }^{37}$

Seppure in maniera disorganica, è però solo con l'articolo 7o., $1^{\circ}$ co., I. 180/1978 che vennero espressamente trasferite alle regioni "le funzioni amministrative concernenti l'assistenza psichiatrica in condizioni di degenza ospedaliera già esercitate dalle province", lasciando a queste ultime la competenza sull'assistenza psichiatrica ambulatoriale e domiciliare, senza peraltro disciplinare le forme del necessario coordinamento organico e funzionale tra le strutture. ${ }^{38}$

Un assetto sistematico alla materia è stato dato dalla legge istitutiva del Servizio Sanitario Nazionale, I. 833/1978, la quale indica all'articolo 10. come compito coordinato dello Stato, delle regioni e degli enti locali territoriali, l'attuazione del complesso delle funzioni, strutture e servizi destinati alla promozione, al mantenimento e al recupero della salute fisica e psichica della popolazione, secondo modalità che garantissero l'eguaglianza dei cittadini ${ }^{39}$. In questa prospettiva appare rilevante la previsione dell'articolo $20 ., 2^{\circ}$ co., lett. g) che stabiliva tra gli obiettivi che le istituzioni avrebbero dovuto perseguire "la tutela della salute mentale,

\footnotetext{
${ }^{37}$ Sulle problematiche connesse al decentramento amministrativo in materia sanitaria si richiama Spadaro Norella, C., “Condizionamento dei poteri regionali in materia di assistenza sanitaria: dalla legge núm. 382 del 1975 alla legge núm. 833 del 1978", Assistenza sanitaria, 1979, p. 235 y ss.; Angiolini, V., “L'amministrazione locale nella riforma sanitaria: spunti per una definizione obiettiva del fenomeno autonomistico", Jus, 1979, p. 232 ss.

${ }^{38}$ Ciò consentì alla province di continuare a gestire l'assistenza psichiatrica extra-ospedaliera, perpetuando le scelte politico-organizzative ormai sedimentate, senza attivare iniziative di rinnovamento in stretta collaborazione con gli organi (ospedali generali) deputati all'assistenza ospedaliera. In tal modo molte amministrazioni provinciali poterono impedire non solo ogni attività decentrata sul territorio, ma anche l'apertura degli stessi servizi speciali di diagnosi e cura presso gli ospedali generali. In tal senso Vecchietti, A. M., op. cit., pp. 206 y ss.

${ }^{39}$ Corasaniti, A., “Commento agli artt. 1 e 2 legge 23 dicembre 1978, n. 833”, Leggi civili commentate, 1979, pp. 1194 y ss.; Casetta, E., “Considerazioni generali sulla legge istitutiva del servizio sanitario nazionale”, Tribunale amministrativo regionale, 1979, t. II, pp. 405 y ss.; in generale Roversi Monaco, F. (a cura di), Il Servizio Sanitario Nazionale. Commento alla legge 23 dicembre 1978, Milano, Giuffrè, núm. 833, 1979.
} 
privilegiando il momento preventivo e inserendo i servizi psichiatrici nei servizi sanitari generali, in modo da eliminare ogni forma di discriminazione e di segregazione, pur nella specificità delle misure terapeutiche e da favorire il recupero e il reinserimento sociale dei disturbati psichici".

Sotto il profilo strettamente ordinamentale, la I. 833/1978 attribuiva, con l'articolo 50., alle regioni le funzioni di indirizzo e coordinamento in materia sanitaria in generale e psichiatrica in particolare, nel rispetto dei criteri di programmazione economica nazionale, nonché, ai sensi dell'articolo 11, le funzioni legislative e amministrative proprie e delegate, seguendo il metodo della programmazione pluriennale, da attuarsi attraverso la predisposizione di piani sanitari regionali.

Risulta essenziale, alla luce dell'ispirazione di fondo della riforma, volta a espandere i servizi psichiatrici di territorio, quanto disposto dall'articolo 34 che demandava alle leggi regionali l'istituzione dei servizi territoriali destinati alla prevenzione, alla cura e alla riabilitazione delle malattie mentali entro strutture dipartimentali, sia di tipo ospedaliero che extra-ospedaliero. L'opera di de-istituzionalizzazione avviata dalla riforma non poteva certo limitarsi all'abbattimento delle strutture manicomiali, dovendo prospettare un percorso alternativo attraverso l'edificazione di un sistema di servizi di prossimità, dotati di mezzi e operatori qualificati, in grado non solo di 'fare' prevenzione ed educazione sanitaria alla popolazione, ma anche di farsi carico del reinserimento sociale delle persone con sofferenze psichiche.

Delineato, seppur sinteticamente, lo sviluppo normativo in materia, si può dire che sul piano attuativo, le Regioni, nel corso degli anni ‘80, non hanno dato seguito alle previsioni della legge del 1978, 'dimenticando' di dotarsi di apposite leggi in materia. ${ }^{40}$ Tuttavia i ritardi nell'istituzione delle Unità sanitarie locali (USL), gli inadeguati finanziamenti statali, i blocchi degli organici regionali resero difficile, al di là delle resistenze culturali e politiche, dare concreta traduzione nella pratica alle nuove normative. ${ }^{41}$ Tutto ciò ha determinato conseguenze che ancora oggi caratterizzano quell'immagine 'a macchia di leopardo' che meglio definisce il livello di presenza

\footnotetext{
${ }^{40}$ La maggior parte delle Amministrazioni regionali peraltro non adottò mai alcun Piano Sanitario Regionale: di fatto, nel corso degli anni Ottanta, essi furono approvati solo in Emilia Romagna, Piemonte, Marche, Valle d'Aosta, Toscana, Umbria, Veneto, Friuli e nella provincia autonoma di Bolzano. Per alcuni momenti di riflessione sulla riforma dei primi anni Ottanta: Misiti, R. et al., La riforma psichiatrica: prima fase di attuazione, Roma, Il Pensiero Scientifico, 1981; Piro, S. y Oddati, A. (a cura di), La riforma psichiatrica del 1978 e il meridione d'Italia: studio sullattuazione della Legge núm.180 del 13 maggio 1978 in sei province dell'Italia meridionale peninsulare, Roma, Il Pensiero Scientifico, 1983.

${ }^{41}$ Come scrive De Vito, C G., I luoghi della psichiatria. Una ricerca sulla rete dei servizi di salute mentale in Toscana a trent'anni dalla legge Basaglia, Firenze, Polistampa, 2010, p. 46. “L'inerzia delle istituzioni e il mancato collegamento con i servizi territoriali fecero del periodo tra il 1983 e il 1993 una fase di «sostanziale abbandono e di lenta trasformazione della struttura». La sintesi di quel cinico disinteresse verso le persone ancora ricoverate negli ospedali psichiatrici era in un neologismo allora molto in voga: «residui manicomiali»".
} 
ed efficienza dei servizi psichiatrici nelle varie aree del nostro Paese: difatti, a fronte di alcune esperienze particolarmente importanti ${ }^{42}$-ove $\mathrm{i}$ processi di deistituzionalizzazione e reinserimento degli infermi di mente figurano assai sviluppati- sussistono situazioni nelle quali i servizi sono assenti, privi di risorse materiali ed umane, perpetuando attraverso la presenza di strutture residenziali di lungo degenza (alle volte in condizioni di profondo degrado) quei manicomi che la legge ‘Basaglia' intendeva cancellare dalla storia del nostro paese.

\section{LA SANITÀ NEL PROCESSO DI DECENTRAMENTO E AZIENDALIZZAZIONE}

I fattori organizzativi, gestionali e quelli tecnici influiscono in maniera ormai determinate nelle strutturazione dei servizi e sulle modalità attraverso le quali le prestazioni, in particolare quelle legale alla salute, vengo ad essere rese disponibili e fruite dai cittadini-utenti. Per tale motivo, nel contesto dell'analisi qui condotta, non poteva essere omesso il riferimento al fenomeno della 'aziendalizzazione ${ }^{43}$ che, a partire dall'inizio degli anni '90, ha travolto e modificato il nostro Servizio Sanitario Nazionale e di riflesso anche le strutture preposte alla tutela della salute mentale.

La politica sanitaria nel corso di quel decennio è stata caratterizzata da una forte spinta al decentramento, nella convinzione da parte del legislatore che l'attribuzione del potere decisionale, e delle conseguenti responsabilità finanziarie, a livello locale avrebbe potuto responsabilizzare maggiormente gli amministratori pubblici verso comportamenti di spesa virtuosi; queste scelte sono state accompagnate anche dalla sperimentazione di nuovi modelli organizzativi maggiormente concorrenziali tra fornitori di prestazioni (pubblici o privati convenzionati) con la finalità di creare una sorta di mercato sanitario regolato. ${ }^{44}$

Così, la "riforma bis" e, cioè, il riordino degli anni 1992/1993, voluto dalla legge delega núm. 421 del 23 ottobre 1992 e attuato con i decreti legislativi 502 del 1992 e 517 del 1993, ha avuto come obiettivo generale quello di garantire ai cittadini di poter accedere su tutto il territorio

\footnotetext{
${ }^{42}$ Sull'esperienza toscana De Vito, C. G., op. cit; sull'esperienza lombarda Re, E. (a cura di), I servizi di salute mentale: storia e organizzazione istituzionale, Azienda Ospedaliera Niguarda, Milano, 2006.

${ }^{43}$ Aziendalizzazione che si concreta nella trasformazione della Usl in aziende pubbliche, nella nomina dei loro direttori (che vengono a sostituire i comitati di gestione) da parte dei vertici regionali con ampio potere discrezionale e nella predisposizione di una forma parziale di separazione tra erogatori e acquirenti delle prestazioni sanitarie.

${ }^{44}$ La complessità di tali trasformazioni istituzionali nel contesto della struttura dei servizi sanitari derivava dalla necessità non solo di governare nuovi profili di una perdurante idea di salute come bene pubblico, ma anche di rispondere a nuove esigenze sociali capaci di incidere sulla tutela delle nuove esigenze emergenti nella popolazione. Sul punto Petretto, A., Lorenzini, S. y Scicolone, N., "Il processo di decentramento sanitario tra passato e futuro", in Fiorentini, G. (a cura di), I servizi sanitari in Italia, Bologna, Il Mulino, 2003, pp. 39 ss.; Crosetti, A., “Il Servizio sanitario. Profili organizzativi", in Ferrara, R. (a cura di), Salute e sanità, Trattato di biodiritto, diretto da S. Rodotà e P. Zatti, Milano, Giuffrè, 2010, pp. 217 y ss.
} 
nazionale ad una gamma di prestazioni di assistenza sanitaria predefinita, assicurando, nello stesso tempo, il rispetto dei vincoli di spesa pubblica determinati prospettivamente in funzione dell'andamento dell'economia nazionale. Con tali provvedimenti si è inoltre determinato di riflesso il superamento del sistema organizzativo precedente, che è passato da un modello di servizio nazionale verticalmente integrato (caratterizzato dalla presenza di un ente, lo Stato, che interviene in più fasi del processo produttivo di beni e servizi sanitari), ad un modello misto ove i beni e servizi sanitari sono opera sia del pubblico che del privato. Si è venuta quindi ad affermare, in un'ottica sussidiaria, l'idea che fosse necessario instaurare un sistema di concorrenza tra istituzioni pubbliche e private in funzione del miglioramento della qualità del servizio offerto e in ossequio al principio della libertà di scelta del cittadino. ${ }^{45}$

I limiti ${ }^{46}$ dei d.Igs. 502 e 517 vengono gradualmente evidenziati e si delineano progressivamente, attraverso dibattiti e confronti, nuovi orientamenti di politica sanitaria, che

${ }^{45}$ Mattioni, A. “Le quattro riforme della sanità. Una lettura sinottica di snodi istituzionali fondamentali”, in Balduzzi, R.
(a cura di), Trent'anni di Servizio sanitario nazionale. Un confronto interdisciplinare, Bologna, Il Mulino, 2010, pp. 297 ss. Secondo cui “Dai provvedimenti di riordino emerge un Servizio —in cui le istituzioni non pubbliche accanto a quelle pubbliche sono acquisite al solo fine di una razionalizzazione territoriale- che continua ad essere governato dalla programmazione e che seleziona prestazioni sanitarie, non necessariamente integrate con quelle socio-assistenziali e non necessariamente unitariamente imputate sulla base di un precostituito fabbisogno finanziario messo a disposizione, alle quali accede l'universalità dei soggetti, ai quali può essere chiesto di partecipare anche finanziariamente".

${ }^{46}$ Così, ad esempio, un effetto tangibile dell'aziendalizzazione sui servizi di salute mentale è dato dal cd. «gigantismo istituzionale», ovvero dalla creazione di servizi dotati di un elevato numero di operatori, strutture e competenti su un ampio bacino d'utenza. Tale condizione ha ingenerato delle conseguenze non controllabili in termini di gerarchizzazione endo-strutturale, di incapacità di razionalizzazione, di burocratizzazione e centralizzazione delle attività non solo amministrative ma anche prestazionali con ricadute sul livello di efficienza del servizio per gli utenti, specie quelli più fragili. La trasformazione dei D.s.m. da strutturali — con gestione di budget autonoma- a tecnicofunzionali, venne a rafforzare il ruolo dei Direttori generali delle Asl in merito all'allocazione delle risorse finanziarie alle Unità funzionali, ora detentrici di budget, favorendo un ulteriore processo di diversificazione tra di esse. Introducendo tetti di spesa e bilanci definiti, l'aziendalizzazione spingeva i responsabili dei servizi a pensare in termini quantitativi, sopravvalutando il peso dei momenti ospedalieri e farmacologici dell'intervento, e sottostimando la rilevazione di molte attività territoriali: ad esempio rispetto ai centri diurni, sede di attività fortemente personalizzate, ma finanziati esclusivamente in base al numero di presenze giornaliere degli utenti. L'ampia e pressoché esclusiva utilizzazione di metodi quantitativi nella valutazione rinviava ad una tendenza riduzionista nella lettura dei bisogni e nelle modalità di intervento, riducendo lo spazio per un'analisi della dimensione soggettiva del disagio mentale e dei meccanismi che determinavano il tipo domanda, che la codificavano in determinate diagnosi e strutturavano l'organizzazione e le modalità della risposta. In questi termini Galli, P. F., “L'aziendalizzazione nei Dipartimenti di salute mentale", Psicoterapia e Scienze umane, 2006, t. 1, pp. 87 y ss.; Piccione, R., Il futuro dei servizi di salute mentale in Italia. Significato e prospettive del sistema italiano di promozione e protezione della salute mentale, Milano, Franco Angeli, 2004, pp. 222 ss.; Scapicchio, P. L. y Trabucchi, M. (a cura di), I servizi psichiatrici nella sanità riformata, Bologna, Il Mulino, 1999, pp. 25 ss. 
trovano verso la fine degli anni ‘90 contestuale espressione nel Piano sanitario nazionale per il triennio 1998-2000 e nella legge 419/98 di delega al Governo per la razionalizzazione del Ssn e nel relativo decreto legislativo.

Per questo con il d.Igs. 229 del 1999 si è inteso riproporre, in una versione razionalizzata, la cultura, le finalità e le idealità proprie della riforma del 1978: ${ }^{47} \mathrm{si}$ inscrive in tale contesto la sussunzione delle attività di tutela della salute entro la categoria di funzione pubblica realizzativa del diritto soggettivo attribuito alla universalità delle persone, di cui si garantisce la libertà e la dignità. Sul piano organizzativo la riforma delinea un sistema sanitario integrato dove le responsabilità programmatorie sono attribuite sia al governo centrale che agli enti locali, definendo in tal modo un modello di cooperazione amministrata. Da questo momento, infatti, le Regioni concorrono alla definizione del Piano sanitario nazionale ${ }^{48}$ e a loro volta i comuni sono responsabili dell'organizzazione dei servizi, attraverso la definizione dei distretti, nella maniera più utile per i cittadini. In questo contesto, in coerenza con lo sviluppo di significative forme di integrazione socio-assistenziale, si viene a strutturare un sistema di governo della salute in cui, sia pure in via embrionale, trovano considerazione anche il ruolo del volontariato e delle espressioni del pluralismo etico-culturale. ${ }^{49}$

Si può concludere sul punto notando come la più rilevante novità delle riforme legislative degli anni '90 sia rappresentata dalla nuova strutturazione dei rapporti tra Stato e Regioni in campo sanitario, in cui la programmazione della politica sanitaria - in virtù del Piano sanitario nazionale, della definizione degli obiettivi fondamentali di prevenzione, cura e riabilitazione, di quella delle linee di indirizzo del SSN - avviene attraverso una procedura di 'concertazione' tra i due livelli di

${ }^{47}$ Giarelli, G., “Convergenza e divergenza nelle riforme dei servizi sanitari nazionali euro-mediterranei”, in Vicarelli, G. (a cura di), Regolazione e governance nei sistemi sanitari europei, Bologna, Il Mulino, 2011, p. 39. Secondo cui “Assieme al II Piano sanitario nazionale 1998-2000 (la riforma operata con il d.lgs. 229 del 1999) avrebbe dovuto rappresentare forse uno dei tentativi più ambiziosi in Europa di creare un quadro di regolamentazione dettagliata, basato sui quattro pilastri strategici dei piani sanitari regionali, del processo di accreditamento istituzionale, della valutazione di qualità delle cure e della promozione di forme di integrazione socio-sanitaria a livello istituzionale, organizzativo e professionale".

${ }^{48}$ Il Piano sanitario nazionale, di impostazione profondamente innovativa e centrata sugli obiettivi di salute, afferma che gli interventi in merito ai determinanti non sanitari della salute (fattori ambientali, sociali, economici, ecc.) richiedono un coordinamento intersettoriale, a livello governativo regionale e locale, che deve tradursi in strategie condivise per obiettivi comuni, e che l'integrazione delle responsabilità e delle risorse rappresenta quindi una condizione essenziale per migliorare l'efficacia degli interventi.

${ }^{49}$ Per tutti Balduzzi, R. y G. Di Gaspare, G. (a cura di), L'aziendalizzazione nel d.Igs. núm. 229/99, Milano, Giuffrè, 2001; Id. (a cura di), Sanità ed assistenza dopo la riforma del Titolo V, Milano, Giuffrè, 2002; Ranci Ortigosa, E., "Il rapporto tra servizi sociali e sanitari dopo la I. n. 328 del 2000', Ragiusan, 2005, pp. 1 y ss.; Pioggia, A., et al., Oltre l'aziendalizzazione del servizio sanitario. Un primo bilancio, Milano, Franco Angeli, 2008. 
governo, centrale e regionale, volta a garantire l'universalità e uniformità delle prestazioni in un contesto razionalizzato.

\section{I PROGETTI OBIETTIVO: DIRETTIVE PER L'ATTUAZIONE DELLA L. 180/1978}

Come già accennato, se la riforma psichiatrica si poneva l'obiettivo della contestualità tra il superamento dell'ospedale psichiatrico e la costruzione dei servizi, proprio a questo livello si determinarono le maggiori difficoltà, con una forte "discrepanza temporale" tra il lavoro di dimissione e la strutturazione dell'intervento sul territorio. ${ }^{50}$ In parte ciò era una conseguenza inevitabile della scelta di rottura fatta dalla legge 180, per la quale l'abolizione degli ospedali psichiatrici segnava un punto di non ritorno, che tuttavia presupponeva la necessaria strutturazione di una rete di servizi territoriali di salute mentale ispirati ad un modello del tutto alternativo a quello di assistenza psichiatrica manicomiale.

Nel contesto del processo di rinnovamento dell'organizzazione sanitaria si inserisce il Progetto Obiettivo "Tutela della salute mentale", approvato con d.P.R. 7 aprile 1994, che ha rappresentato, dopo 16 anni dall'approvazione della legge 180, la prima regolamentazione a livello nazionale volta a dettare i principi direttivi e i criteri guida per strutturare in maniera tendenzialmente omogenea il sistema dei servizi per la salute mentale sul territorio nazionale. Nelle premesse del documento si da atto del fatto che la legge 180 abbia

...postulato un diverso approccio alla malattia mentale, modificando gli obiettivi fondamentali dell'intervento pubblico dal controllo sociale dei malati di mente alla promozione della salute e alla prevenzione dei disturbi mentali e spostando l'asse portante delle istituzioni assistenziali dagli interventi fondati sul ricovero ospedaliero a quelli incentrati sui servizi territoriali.

Tuttavia proprio il risultato non soddisfacente dell'attuazione della riforma, la scarsa articolazione dei servizi, nonché il ritardo nel predisporre strutture ed interventi sul territorio, con un sovraccarico funzionale per le famiglie dei malati, hanno spinto l'esecutivo a definire linee uniformi di sviluppo strutturali del sistema. ${ }^{51}$ In tal senso, il progetto prevedeva la creazione di Dipartimenti di salute mentale (D.S.M.) corrispondenti al bacino di utenza e ai bisogni a livello

\footnotetext{
${ }^{50}$ Il processo di riforma non poteva affermarsi in pochi anni, anzi, col passare del tempo, le dimissioni dei ricoverati negli ospedali psichiatrici diventavano sempre più complesse a mano a mano che si trattava di riportare sul territorio pazienti cronicizzati da decenni di ricovero. All'affermazione del principio della deistituzionalizzazione dovevano pertanto seguire lunghi periodi di riabilitazione e risocializzazione, sia dentro che fuori dall'ospedale psichiatrico, affinché quelle persone potessero riacquisire un minimo di autonomia e di fiducia in se stessi.

${ }^{51}$ In tal senso la trattazione, contenuta nel Progetto Obiettivo, è stata interamente concentrata sulle caratteristiche tecnico-organizzative del Dipartimento di salute mentale, senza riferimenti alla realtà più ampia dei servizi sociosanitari e tantomeno alla partecipazione della società civile.
} 
regionale e che, in ogni Dipartimento di salute mentale (D.S.M.), la struttura ordinaria comprendesse i seguenti Servizi: a) Centri di salute mentale; b) Servizi di diagnosi e cura (S.P.C.D.); c) strutture semiresidenziali (day hospital psichiatrico e centro diurno); $d$ ) strutture residenziali, specie in considerazione di bisogni di lungo-assistenza, la domanda di residenzialità protetta e semiprotetta.

Il rilancio del processo riformatore della 180 coincide dunque con un ulteriore spostamento del baricentro dei servizi di salute mentale in direzione di un Dipartimento "forte", connotato in senso specialistico, che si viene ad inserire in un modello organizzativo nel quale la protezione della salute mentale è garantita dall'unitarietà e integrazione dei servizi psichiatrici che insistono sul territorio e soprattutto dall'azione congiunta e coordinata tra tali servizi e quelli socio-sanitari confinanti in termini di competenze (Consultori, Sert ecc.).

Successivamente, con il Progetto obiettivo nazionale sulla tutela della salute mentale 19982000 (d.P.R. 1 novembre 1999), nell'individuare la salute mentale fra le tematiche ad elevata complessità per le quali si ritiene necessaria l'elaborazione di specifici atti di indirizzo, si è preso atto e si è tentato di colmare le lacune che connotavano il precedente progetto: infatti, se da una parte si può affermare che, pur con differenti gradi di realizzazione, il modello dipartimentale era stato avviato e che la de-ospedalizzazione era un fatto quasi compiuto, dall'altra parte la salute mentale in età evolutiva (nelle forme domiciliari, ambulatoriali, semiresidenziali, residenziali e ospedalieri) continuava ad offrire servizi carenti, permanevano inoltre lo scarso coordinamento e la conflittualità tra le varie figure professionali e non si era riusciti ad implementare le reti informali di solidarietà creando una collaborazione con il terzo settore e le famiglie.

Il Progetto Obiettivo 1998-2000 ha perciò formulato ulteriori cinque "obiettivi di salute", tuttora attualissimi: a) promuovere la salute mentale nell'intero ciclo della vita, dall'età evolutiva all'anziano; b) perseguire la prevenzione, sia primaria che secondaria, del disagio giovanile; c) salvaguardare la qualità di vita del nucleo familiare del paziente; d) ridurre il numero ancora elevato di suicidi e tentati suicidi; e) ricostruire il tessuto affettivo, sociale e relazionale del paziente. $^{52}$

Elemento caratterizzante del Progetto Obiettivo è rappresentato dalla programmazione dei progetti terapeutici e di risocializzazione, dall'incremento degli interventi della rete sociale e familiare, oltre che dalla limitazione della cronicità e delle pratiche assistenzialistiche. ${ }^{53}$

\footnotetext{
${ }^{52}$ Per maggiori approfondimenti si rinvia ai lavori del Centro Studi del Ministero della Sanità (in collaborazione con il Centro studi Labos) e dell'Istituto Italiano di Medicina Sociale AA. VV., L'assistenza psichiatrica in Italia. La normativa e la diffusione dei servizi sul territorio. Progetto Obiettivo "Tutela della Salute mentale", Roma, Ter edizioni, 1998; AA. VV., Utenza psichiatrica e servizi, Roma, Ter edizioni, 1998.
}

${ }^{53}$ Viani, G. y Tiberio, A., Manuale di legislazione sanitaria, Milano, Franco Angeli, 2002, pp. 169 y ss. 
È interessante notare, sotto il profilo dei principi costituzionali adombrati nei summenzionati obiettivi, come ne emerga una forte impronta personalista rivolta a tutelare l'uomo concreto nei suoi aspetti esistenziali più profondi: in particolare il richiamo alla necessità di ricostruire il tessuto affettivo, sociale e relazionale del paziente intreccia tali tematiche con l'affermazione, anche in questo campo, del principio di dignità, che si traduce in un efficace strumento di reazione all'isolamento del fenomeno giuridico in una visione astratta, tecnicista e neutralista, nella quale sfuma ogni senso di morale coinvolgimento e responsabilità.

Nel campo della salute mentale il riconoscimento della dignità come valore della persona può essere declinato in una duplice prospettiva plurale e soggettiva: ${ }^{54}$ inteso come riconoscimento dell'inviolabilità (e autonomia) della stessa, e al contempo, in funzione del richiamo alla 'pari dignità sociale', come strumento per ridare spessore alla cittadinanza delle persone con infermità mentali, laddove, sancita la loro libertà formale (anche attraverso le previsioni della I. núm. 833/1978), si rende necessario garantire e approntare i mezzi atti rimuovere gli ostacoli che ne impediscono la piena realizzazione.

La positivizzazione del principio di dignità fa sì che la persona, considerata all'interno di una dimensione relazionale, venga iscritta in un orizzonte di irrinunciabile appartenenza all'umanità, intesa come tessuto di mutue relazioni fondate su di un imperativo di reciproco riconoscimento: essa viene dunque compresa come categoria del «prossimo», della comunanza con l'altro che è propria di ogni individuo, quale als Mitmensch, ossia come punto di riferimento di una relazione normativa con se stesso e con l'insieme della umanità. Citando Hofmann, ${ }^{55}$ la dignità non può essere pensata svincolata da una comunità concreta di riconoscimento e significazione. In tale prospettiva l'etica dei diritti discende, così, da un dovere originario, quello di riconoscere l'altro e i suoi diritti, nonché quello di esercitarli in modo da non far venir meno il rispetto di sé.

Vi è da segnalare inoltre come, per la prima volta ufficialmente, viene affrontato in modo organico il problema della prevenzione e promozione della salute mentale, con interventi specifici e precoci sulle condizioni di disagio e disturbo mentale, realizzati anche attraverso lo strumento del 'patto della salute' volto a coordinare ed integrare le agenzie formali ed informali in grado di contribuire alla costruzione di un progetto di salute mentale di comunità.

\footnotetext{
${ }^{54}$ Il concetto di dignità, in quest'ultima accezione, socializza il rapporto di sé a sé, trasformandolo in un rapporto di sé con gli altri (l'umanità): così nel rapporto che l'individuo sviluppa con sé stesso (e con il proprio corpo) la dignità opera come sinonimo di libertà e autodeterminazione nella definizione di ciò che è degno o meno; mentre nel rapporto con $\mathrm{i}$ terzi tale concetto opera come dovere di rispettare l'autonomia e le determinazioni altrui.

${ }^{55}$ Hofmann, H., “Die versprochene Menschenwürde”, Archiv des öffentlichen Rechts, 1993, 118, pp. 353 y ss.; trad. de “La promessa della dignità umana”, Rivista internazionale di filosofia del diritto, 1999, pp. 620 y ss.
} 
La fase di attuazione del secondo Progetto ha consentito di affrontare diversi problemi organizzativi e strutturali relativi all'offerta di prestazioni di cura ed assistenziali: ciò è avvenuto attraverso lo sviluppo, non sempre programmato, del settore residenziale, un notevole sforzo di qualificazione degli interventi mediante gli strumenti del miglioramento continuo e dell'accreditamento ed il ruolo molto importante assunto dall'associazionismo, dalle pratiche di auto-mutuo aiuto con il coinvolgimento degli utenti e familiari nei momenti di programmazione, monitoraggio e verifica delle politiche e dei progetti.

Un altro aspetto saliente, specie ai nostri fini, è dato dalla previsione nel Progetto della necessità di implementare strumenti di buona pratica clinica (Ebp/Ebn), che tengano conto del recepimento di linee guida e delle migliori evidenze disponibili ${ }^{56}$.

L'adozione dei Progetti Obiettivo ha costituito quindi un catalizzatore per l'approvazione da parte delle regioni di apposite discipline normative (leggi e regolamenti), piani attuativi e progetti obiettivo regionali, finalizzati a definire sia la dimensione organizzativa-gestionale che quella clinica ${ }^{57}$. Tuttavia, a fronte di una parziale uniformità della disciplina di dettaglio, la complessità dell'assetto gestionale e organizzativo operante nelle diverse realtà regionali ha determinato una strutturazione diversificata dei servizi il che ha posto in evidenza l'esigenza di un governo del sistema in grado di considerare il ruolo, le funzioni e le competenze dei diversi soggetti e Enti coinvolti. ${ }^{58}$ Le diverse regolamentazioni hanno poi introdotto la metodologia dei percorsi clinici, garantendo agli utenti una valutazione approfondita delle loro esigenze e percorsi di cura differenziati e hanno identificato modelli clinico-organizzativi per governare il processo assistenziale. $^{59}$

\footnotetext{
${ }^{56}$ Su cui si tornerà per approfondire l'argomento nel §. 7.

${ }^{57}$ Marino, F., “La tutela della salute mentale nelle leggi regionali e nei progetti-obiettivo nazionali”, in Scapicchio, P. L. y Trabucchi, M., op. cit., pp. 33 y ss.

${ }^{58}$ In tal senso è stato notato come, dopo il 1978, si sia verificato un processo di regionalizzazione della cura della salute mentale, creando 21 sistemi regionali di salute mentale, molto diversi in termini di organizzazione, rete di servizi, accessibilità, modalità di cura. Lora, A., "An overview of the mental health system in Italy", Annali Istituto Superiore di Sanità, 2009, 45, 1, pp. 5 y ss.; De Girolamo, G. et al., "The current state of mental health care in Italy: problems, perspectives, and lessons to learn", Eur. Arch Psychiatry Clin Neurosci, 2007, 257, pp. 83 y ss.

${ }^{59}$ Si deve sottolineare come denominatore comune degli interventi nazionali e regionali, rispetto all'obiettivo di indicare i modelli e gli strumenti per sviluppare una nuova policy per la salute mentale, sia la consapevolezza che la risposta ai bisogni delle persone non può essere trovata solo nell'ambito sanitario, ma necessariamente deve coinvolgere anche l'ambito sociale e considerare l'aspetto esistenziale e relazionale della vita dell'uomo. In questa prospettiva diviene centrale il tema dell'integrazione e del collegamento tra i diversi soggetti, istituzionali e non istituzionali, coinvolti nella tutela della salute mentale: ad essi, nel rispetto dei reciproci ruoli e funzioni, in una logica di sussidiarietà, viene chiesto di partecipare a una "comunità per la salute mentale" assumendosi la propria parte di
} 
Vi è infine da segnalare l'approvazione, in data 24 gennaio 2013, da parte della Conferenza unificata Stato-Regioni ed Enti locali del "Piano di azioni nazionale per la salute mentale", che si fonda sulla necessità di lavorare per progetti di intervento specifici e differenziati sulla base della valutazione dei bisogni delle persone e della implementazione di percorsi di cura che sappiano intercettare le attuali domande di salute e contribuiscano a rinnovare l'organizzazione dei servizi, le modalità di lavoro delle equipe, i programmi clinici aggiornati offerti agli utenti. Il documento delinea un modello di approccio alle problematiche e ai bisogni emergenti nel settore della salute mentale che intende garantire: a) accessibilità, presa in carico, continuità delle cure e personalizzazione del progetto; $b$ ) percorsi a differente intensità assistenziale in rapporto ai bisogni di cura; $c$ ) servizi flessibili, orientati sui bisogni delle persone; $d$ ) Lea garantiti dalla Asl nel suo complesso e non solo dal DSM o dai servizi per i disturbi neuropsichici in infanzia e adolescenza; e) percorsi esigibili individualmente, anche quando inseriti in attività di gruppo o in attività comunitarie.

Ciò conferma la necessità che i percorsi di cura debbano essere realizzati da parte dei servizi specialistici per la salute mentale (DSM e/o servizi per i disturbi neuropsichici dell'infanzia e dell'adolescenza) privilegiando una metodologia "dal basso", per valorizzare le buone pratiche esistenti a livello locale, oltre che regionale, favorendo al contempo il confronto con le reti di sostegno formali ed informali.

\section{I LEA E LA SALUTE MENTALE: LIMITI E SFIDE FUTURE}

Vi è uno stretto legame tra processo di regionalizzazione, adozione dei Progetti Obiettivo e definizione dei Livelli essenziali di assistenza (LEA), che viene ad involgere cambiamenti maggiori, incidenti sull'impalcatura istituzionale, sulle basi cognitive delle istituzioni, sulle grammatiche di giustizia e sui modi in cui le norme sociali sono istituite e praticate; e che inevitabilmente toccano anche il diritto nel suo insieme. ${ }^{60}$

La clausola dei "Iivelli essenziali delle prestazioni concernenti i diritti civili e sociali che devono essere garantiti su tutto il territorio nazionale" (cd. LEP) di cui all'articolo $117,2^{\circ}$ co., lett. m) della Costituzione ${ }^{61}$ rappresenta, in tal senso, uno strumento di analisi privilegiata dei cambiamenti che hanno interessato i diritti sociali, nel loro aspetto statico e dinamico, e il delicato rapporto tra

compiti e di responsabilità. G. Biffi, G. y De Isabella, G., Nuove sfide per la salute mentale: innovazioni cliniche e organizzative, Santarcangelo di Romagna, Maggioli, 2012.

${ }^{60}$ De Leonardis, O., "Verso un equilibrio dei legami sociali? Sguardi obliqui sulle metamorfosi della penalità”, Studi sulla questione criminale, 2009, 1, p. 15.

${ }^{61}$ Da rammentare che all'articolo 120 Costituzione, al secondo comma si riconoscono al Governo poteri sostitutivi in presenza di diverse circostanze, tra cui quella della "tutela dei livelli essenziali delle prestazioni concernenti i diritti civili e sociali". 
i soggetti che, ai vari livelli, ne devono garantire la tutela e l'attuazione. ${ }^{62}$ Sotto il profilo ordinamentale, è bene rammentare che la determinazione dei LEP rappresenta un ambito attribuito alla competenza legislativa esclusiva dello Stato, ${ }^{63}$ mentre la "tutela della salute" rientra nel novero delle materie oggetto di potestà concorrente Stato-regioni, elencate nell'articolo 117, $3^{\circ}$ co., Costituzione. ${ }^{64}$ Peraltro, come si è sottolineato, ${ }^{65}$ la peculiarità propria del contesto materiale dei LEP

...consisterebbe nella definizione teleologica di ambiti valoriali, nell'enunciazione di finalità che attraversano orizzontalmente l'ordinamento, coinvolgendo interessi disparati o conglobando oggetti dispersi nell'ordine formale delle competenze. La trasversalità di questi ambiti riservati alla competenza esclusiva del legislatore statale sarebbe dotata di una forte capacità espansiva, tale da investire anche materie di competenza regionale, sia concorrente che residuale.

Anche in virtù di tale tendenza espansiva si è attribuita alla clausola dei livelli essenziali una funzione di unificazione nell'ambito del sistema istituzionale policentrico, atta ad comprendere la

${ }^{62}$ D'Aloia, A., “Diritti e Stato autonomistico. Il modello dei livelli essenziali delle prestazioni”, Le Regioni, 2003, pp. 1064 y ss.; Balboni, E., "Il concetto di 'livelli essenziali e uniformi' come garanzia in materia di diritti sociali”, Le Istituzioni del Federalismo, 2001, pp. 1109 y ss.; anche Guiglia, G., I livelli essenziali delle prestazioni sociali alla luce della recente giurisprudenza costituzionale e dell'evoluzione interpretativa, Giuffrè, Milano, 2007, pp. 15 y ss.

${ }^{63}$ Secondo quanto sancito dalla Corte costituzionale nella sentenza núm. 282/2002, “non si tratta di una 'materia' in senso stretto, ma di una competenza del legislatore statale idonea ad investire tutte le materie, rispetto alle quali il legislatore stesso deve poter porre le norme necessarie per assicurare a tutti, sull'intero territorio nazionale, il godimento di prestazioni garantite, come contenuto essenziale di tali diritti, senza che la legislazione regionale possa limitarle o condizionarle". Altresì Corte Costituzione, sentt. núms. 322/2009 y 10/2010.

${ }^{64}$ La questione è chiaramente molto più complessa: “a dieci anni dall'entrata in vigore della novella costituzionale del 2001 il modello relazionale dell'allocazione delle competenze normativo/legislative fra lo Stato e le Regioni nel campo della sanità è ben lungi dall'essere stabilizzato e, soprattutto pacificato, essendo invece sotto gli occhi di tutti i guasti di un contenzioso alluvionale che si focalizza principalmente attorno alle reciproche ragioni del dare e dell'avere. Il che è sicuramente anche causato da una certa ambigua 'leggerezza' delle norme costituzionali di riferimento, e forse in primo luogo proprio dall'istituto, certo affascinante e suggestivo, della chiamata in sussidiarietà in quanto foriero di una riscrittura per così dire continua e permanente (rebus sic stantibus, in relazione all'attualità degli interessi pubblici puntuali che debbono essere curati) dell'ordine delle competenze dei soggetti del sistema multilivello. Il quadro complessivo si caratterizza, in altre parole, per un'intrinseca, e quasi oggettiva, relativizzazione dei poteri e delle attribuzioni, quasi in sintonia con le regole ordinatrici dei contemporanei modelli di multilevel governance'. Ferrara, R., "Rapporti Stato-Regioni in materia di sanità", in AA. VV., II libro dell'anno del diritto 2012, Roma, Treccani, 2012, pp. 377 y ss.; Carpani, G. y D. Morana, D., “Le competenze legislative in materia di “tutela della salute”, in Balduzzi, R. y Carpani, G. (a cura di), Manuale di diritto sanitario, Bologna, Il Mulino, 2013, pp. 126 y ss.

${ }^{65}$ Pesaresi, E., “La «determinazione dei livelli essenziali delle prestazioni» e la materia «tutela della salute»: la proiezione indivisibile di un concetto unitario di cittadinanza nell'era del decentramento istituzionale", Giurisprudenza costituzionale, 2006, p. 1734. 
pluralità delle situazioni giuridiche soggettive che costituiscono il fascio dei diritti del moderno concetto di cittadinanza sociale.

Si comprende allora perché il legislatore ordinario, ${ }^{66}$ in dialogo con gli strumenti di pianificazione nazionale, ${ }^{67}$ abbia qualificato come essenziali quei livelli necessari (e cioè le tipologie di assistenza, i servizi e le prestazioni sanitarie che presentano, per specifiche condizioni cliniche o di rischio, evidenze scientifiche di un significativo beneficio in termini di salute, a livello individuale e collettivo, a fronte delle risorse impiegate) e appropriati (rispetto sia alle specifiche esigenze di salute del cittadino, sia alle modalità di erogazione delle prestazioni), che dunque definiscono, in sanità, il tetto massimo, il livello massimo —non la soglia, cioè il livello minimo— delle garanzie offerte a tutti i consociati.

Sembra quindi che, attraverso la previsione costituzionale, si sia voluto chiarire come l'obiettivo prefissato con la definizione dei livelli essenziali non sia tanto quello di individuare i livelli minimi di assistenza o, addirittura, di individuare i soli servizi che devono essere forniti, ${ }^{68}$ ma piuttosto sia quello di assicurare una comune cittadinanza di diritti su tutto il territorio, a prescindere dal luogo di residenza. In altre parole, la previsione costituzionale sembra adattarsi alle necessità di un assetto istituzionale tendenzialmente federale, in cui, a fronte di 'livelli' di differenziazione fisiologica, si garantiscono standards uniformi relativi alla soddisfazione di bisogni ritenuti particolarmente meritevoli di tutela a prescindere dall'appartenenza territoriale amministrativa, in quanto espressione di un'esigenza di uguaglianza tra i cittadini a cui il nostro ordinamento non può rimanere indifferente. ${ }^{69}$

Da questa esigenza emerge un ultimo profilo che connota la previsione costituzionale dei livelli essenziali, ovvero la valenza vincolante degli stessi, nella misura in cui non costituiscono un

\footnotetext{
${ }^{66}$ Articolo 1o., $7^{\circ}$ co., d.lgs. núm. 502/1992 e s.m.i.

${ }^{67}$ D.P.R. 23 luglio 1998, approvazione del Piano sanitario nazionale 1998-2000.

${ }^{68}$ Sulla distinzione tra approccio 'minimalista' e 'massimalista' adottato dalla dottrina nell'interpretazione della clausola si veda Trucco, L., "Livelli essenziali delle prestazioni e sostenibilità finanziaria", Atti del Convegno annuale dell'Associazione 'Gruppo di Pisa' dedicato a “I diritti sociali: dal riconoscimento alla garanzia. Il ruolo della giurisprudenza", in www.gruppodipisa.it, 2.

${ }^{69}$ In dottrina, si è parlato pertanto dei livelli essenziali come del “nuovo nome dell'uguaglianza”. Balboni, E., “Livelli essenziali. Il nuovo nome dell'uguaglianza? Evoluzione dei diritti sociali, sussidiarietà e società del benessere", in Balboni, E. et al., (a cura di), Il sistema integrato dei servizi sociali, Milano, Giuffrè, 2003, pp. 27 ss. Per usare le parole della Corte costituzionale (sent. núm. 10/2010), si tratta di “un fondamentale strumento per garantire il mantenimento di una adeguata uniformità di trattamento sul piano dei diritti di tutti i soggetti, pur in un sistema caratterizzato da un livello di autonomia regionale e locale decisamente accresciuto".
} 
mero orientamento programmatico, ma pongono vincoli precisi ${ }^{70}$ che circoscrivono l'ambito d'azione dei soggetti istituzionali deputati alla fornitura dei servizi a fronte dei quali si ergono diritti che possono essere immediatamente azionati, anche a prescindere dall'assetto organizzativo predisposto dalle amministrazioni. ${ }^{71}$

All'indomani dell'entrata in vigore del nuovo Titolo $V$ della Costituzione, venivano definiti dal governo, d'intesa con la Conferenza permanente per i rapporti tra lo Stato, le regioni e le province autonome di Trento e Bolzano (accordo del 22 novembre 2001), attraverso il d.P.C.m 29 novembre $2001{ }^{72}$ i livelli essenziali di appropriatezza delle prestazioni sanitarie e dei servizi che il Sistema Sanitario Nazionale si impegna a fornire a tutti i cittadini. ${ }^{73}$

Sulla base di quanto prescritto nel citato decreto il Servizio sanitario nazionale assicura i livelli di assistenza (LEA), ovvero le prestazioni, definite nelle loro caratteristiche tipologiche, quantitative e qualitative, che il servizio pubblico è tenuto a garantire su tutto il territorio nazionale, gratuitamente $o$ in regime di compartecipazione.

${ }^{70}$ In sostanza si può sostenere che la nozione di essenzialità dei livelli delle prestazioni si componga di una parte essenziale ed una sfera accessoria, la quale sola può prestarsi a differenti qualificazioni e ricevere una differente intensità di tutela. In questa prospettiva, sembra che la determinazione dei livelli essenziali presupponga, prioritariamente, l'identificazione degli interessi che si intendono tutelare, indisponibili per il legislatore regionale, e successivamente la scelta in ordine alle modalità di tutela, profilo, quest'ultimo direttamente incidente sul versante del contenuto del diritto, pur se suscettibile di essere diversamente e più intensamente assicurato a livello locale. L'essenzialità consiste, allora, nella garanzia del riconoscimento di uguali condizioni di godimento dei diritti, pur senza pregiudicare la scelta di una differenziazione in melius nei diversi contesti regionali, nel rispetto della soglia stabilita dal livello centrale di governo, per tutto il territorio nazionale. Pesaresi, E., op. cit., p. 1743.

${ }^{71}$ Sulla giustiziabilità dei LEP si veda Gambino, S., “Diritto alla vita, libertà di morire con dignità, tutela della salute. Le garanzie dell'articolo 32 della Costituzione", in www.forumcostituzionale.it, pp. 7-9.

${ }^{72}$ Si rammentano le limitate innovazioni apportate dal d.P.C.m. 28 novembre 2003 e 5 marzo 2007 con riferimento alle problematiche della non autosufficienza. Da ultimo, in base all'Intesa Stato-Regioni del 5 ottobre 2006 “Patto sulla salute" e alla legge 27 dicembre 2006, núm. 296, i LEA sarebbero stati ridefiniti dal d.P.C.m. 23 aprile 2008 poi revocato nell'autunno 2008 per i rilievi sollevati dalla Corte dei Conti per la mancata copertura finanziaria di alcune prestazioni aggiuntive. Si è quindi in attesa dell'adozione di un d.P.C.m. attuativo dell'articolo 5o., d.I. 13 settembre 2012, núm. 158, convertito con modificazioni dalla I. 8 novembre 2012, núm. 189, che prevede l'aggiornamento dei livelli essenziali di assistenza con prioritario riferimento alla riformulazione dell'elenco delle malattie croniche, delle malattie rare, ed inoltre delle prestazioni di prevenzione, cura e riabilitazione rivolte alle persone affette da ludopatia.

${ }^{73}$ Sui LEA si rinvia Molaschi, V., "I livelli essenziali delle prestazioni nella sanità”, in Ferrara, R. (a cura di), Salute e sanità, Trattato di biodiritto, cit., pp. 445 y ss.; Balduzzi, R., “Livelli essenziali e risorse disponibili. La sanità come paradigma”, in Roversi Monaco, F. y C. Bottari, C. (a cura di), La tutela della salute tra garanzie degli utenti ed esigenze di bilancio, Santarcangelo di Romagna, Maggioli, 2012, pp. 79 y ss.; Longo, N., I livelli essenziali delle prestazioni quale clausola di omogeneità sul territorio nazionale, Roma, Aracne, 2012; Messineo, D., La garanzia del "contenuto essenziale" dei diritti fondamentali, Dalla tutela della dignità umana ai livelli essenziali delle prestazioni, Torino, Giappichelli, 2012. 
Quindi nel d.P.C.m. del 2001 vengono elencate, negli allegati, le attività e le prestazioni incluse nei Livelli, le prestazioni escluse, le prestazioni che possono essere fornite dal Servizio sanitario nazionale solo a particolari condizioni, dovendosi precisare che «dell'offerta concreta fanno parte non solo la qualità e quantità delle prestazioni che debbono essere assicurate sul territorio, ma anche le soglie di accesso, dal punto di vista economico, dei cittadini alla loro fruizione» (così la sent. 187/2012, riportando testualmente un inciso dalla sent. 203/2008). Ciò in quanto l'equivalenza delle precondizioni e delle modalità di accesso alla fruizione dei servizi, riflette un concetto di cittadinanza sottratto alle dinamiche e alle variabili dei processi di differenziazione, pur necessari, del contesto istituzionale pluralistico attuale.

Seppure "la regolamentazione dell'assetto organizzativo e gestorio degli enti preposti all'erogazione delle prestazioni stesse" non possa costituire un LEP (Corte Costituzione, núm. 387/2007; núm. 166/2008; núm. 207/2010), ${ }^{74}$ vi è una forte correlazione tra i profili erogativi e quelli più prettamente organizzativi, nella misura in cui solo un'efficiente organizzazione è in grado di incidere positivamente sugli obiettivi preposti ed i compiti posti in essere a tutela degli utenti.

Vi è tuttavia da rilevare, in senso critico, come il d.P.C.m. sui LEA sia 'nato già vecchio', non rispecchiando le innovazioni apportate dalla concomitante modifica del Titolo $\mathrm{V}$ della Costituzione e pertanto risultando fortemente condizionato dall'impostazione che connotava il sistema sanitario pre-riforma. In secondo luogo, il decreto descrive i LEA prevedendo un'articolazione dell'assistenza sanitaria in livelli, aree (sanitaria collettiva, distrettuale ed ospedaliera), servizi, prestazioni e procedure, ${ }^{75}$ ma nel definire l'elenco analitico delle prestazioni non ne indica in maniera adeguata i criteri di selezione e controllo da valutarsi in conformità ai principi di efficacia e di appropriatezza. ${ }^{76}$ Inoltre la stessa definizione di standard nazionali (linee

\footnotetext{
${ }^{74}$ Come ricorda la Corte non devono essere confusi “i livelli essenziali di assistenza, che attengono all'erogazione dei servizi in campo sanitario, e le strutture che strumentalmente vengono predisposte per la prestazione dei servizi stessi" (Corte Costituzione núm. 99/2009).

${ }^{75}$ Tenuto conto che, in quanto ascrivibili ad una pluralità di settori, i livelli di prestazione da determinare sfuggono ad una catalogazione omogenea ed i caratteri che li accomunano possono esprimersi solo in termini generali e sotto forma di "princìpi".

${ }^{76}$ Così, ad esempio, durante un ricovero ospedaliero appropriato, un'azienda sanitaria può erogare servizi, prestazioni e procedure inefficaci e inappropriate che consumano preziose risorse. Sul punto le notazioni critiche di Tubertini, C., Pubblica amministrazione e garanzia dei livelli essenziali delle prestazioni. Il caso della tutela della salute, Bologna, Bonomia University Press, 2008, pp. 284-285; Pizzolato, F., “La procedura di determinazione dei livelli essenziali di assistenza sociale", in wWw.amministrazioneincammino.luiss.it, p.14.
} 
guida, report di HTA e Horizon Scanning), che avrebbero dovuto supportare tecnicamente la verifica dei LEA, soprattutto a livello di prestazioni, non è mai decollata. ${ }^{77}$

Dato per scontato infine che i livelli essenziali di assistenza non possono essere considerati come un dato storicamente immutabile, richiedendosi, al contrario, continui aggiornamenti conformemente sia all'evolversi delle condizioni di salute e ai bisogni sanitari della popolazione che al miglioramento delle conoscenze scientifiche, sembra che il procedimento che ne doveva garantire il continuo e progressivo adeguamento non sia stato in grado di garantire la corrispondenza tra il rinnovamento del dato normativo e l'emergere di nuovi bisogni nella realtà sociale.

Nel contesto della salute mentale in particolare, la risposta data dai LEA non ha corrisposto, almeno in parte, alle esigenze determinate sia dalla trasformazione della domanda da parte dell'utenza, sia dalla limitazione delle possibilità di risposta da parte dei servizi psichiatrici pubblici. Carenze che si sono aggravate in corrispondenza con il processo di marginalità sociale crescente nella popolazione in generale, e in particolare tra i soggetti fragili, che costituisce un'emergenza per l'attività dei servizi i quali, strutturati sul modello organizzativo dei servizi di comunità, si trovano di fronte alla necessità di accogliere, oltre alla problematiche connesse alle classiche forme di disturbo psichico, anche la dimensione emotiva della sofferenza collegata alla perdita di disponibilità, di status e di ruolo. ${ }^{78}$

All'aumento della domanda, conseguente ai fenomeni di crescente indebolimento della capacità delle agenzie sociali di dare risposte, si è sovrapposta la riduzione delle risorse umane e materiali. Questo ha determinato una difficoltà reale da parte dei servizi a rispondere in modo integrato, complessivo e continuativo ai bisogni della popolazione, aprendo la strada, non di rado, ad interventi d'urgenza o di semplice consulenza di tipo "cosmetico".

A sua volta, la concomitante perdita (o inefficiacia) di priorità non più corrispondenti al dato reale ha generato ulteriori criticità riguardo alla programmazione degli interventi che richiedono invece una logica integrata di tipo socio-sanitario.

Chiaramente il procrastinare ulteriormente una sistematica strategia di interventi dedicati a queste problematiche (anche in conseguenza della mancata revisione dei LEA) ha comportato

\footnotetext{
77 Lacune che nè il sistema di garanzia per il monitoraggio dell'assistenza sanitaria erogata nelle Regioni italiane, istituito con il d.Igs. 56/2000 (implementato dal d.m. 12 dicembre 2001), né Comitato permanente per la verifica dell'erogazione dei Livelli essenziali di assistenza, istituito presso il Ministero della salute (d.m. 25 novembre 2005) hanno potuto sin'ora colmare. Zocchetti, C., "I livelli di assistenza (LEA): prima parte di un racconto in 3 puntate”, Statistica e Società, 2013, t. 2, pp. 31 y ss.

${ }^{78}$ Sul punto Bauman, S., "Salute e disuguaglianze", in Id., Cose che abbiamo in comune. 44 lettere dal mondo liquido, Bari-Roma, Laterza, 2012, pp. 91 y ss.
} 
non solo il venir meno delle garanzie d'erogazione dei dovuti livelli assistenziali di cura ad una fascia crescente della popolazione ma anche ad una dispendiosa mobilitazione delle poche risorse a disposizione, finalizzandole eminentemente al mero tamponamento delle emergenze che ne conseguono, in primis quelle riconducibili alla non adesione ai piani di cura che è particolarmente sviluppata nella fascia di utenza più marginale.

Tornando al dato normativo, si sottolinea come, nell'ambito del d.P.C.m. del 2001, l'attività sanitaria e socio-sanitaria a favore delle persone con problemi psichiatrici e delle loro famiglie sia stata iscritta, da un lato, nel contesto dell'assistenza territoriale, ambulatoriale e domiciliare, e dall'altro, nell'ambito dell'assistenza semi-residenziale o residenziale -in conformità a quanto già prescritto nel Progetto Obiettivo del 1998- prevedendosi corrispondentemente l'accesso a prestazioni ambulatoriali e riabilitative presso il domicilio o in strutture residenziali o semiresidenziali. $^{79}$

È bene rammentare che il sistema di protezione sociale italiano si basa sull'interazione tra sfera sanitaria e assistenziale, nella misura in cui la risoluzione di determinate problematiche sociali (in senso lato come può essere il disturbo psichico) richiedono un approccio omnicomprensivo, sanitario ed assistenziale, in cui interventi e strategie dell'uno e dell'altro settore siano coordinati e si completino vicendevolmente. ${ }^{80}$ La natura del bisogno, specie in relazione alla condizione di soggetti fragili, è determinata tenendo conto degli aspetti inerenti alle funzioni psicofisiche, alla natura delle attività del soggetto e relative limitazioni, alle modalità di partecipazione alla vita sociale e, infine, ai fattori di contesto ambientale e familiare che incidono sulla risposta al bisogno e sul suo superamento. ${ }^{81}$

\footnotetext{
${ }^{79}$ Il percorso territoriale di un paziente con difficoltà di funzionamento personale e sociale, con bisogni complessi, ivi comprese problematiche connesse a comorbidità somatica, e con necessità di interventi multi professionali, si sviluppa attraverso una presa in carico da parte del Centro di salute mentale (CSM) che elabora un Piano di trattamento individuale (PTI); nell'ambito di detto piano, dando comunque preferenza all'approccio domiciliare e/o ambulatoriale, può essere previsto l'invio e il temporaneo inserimento in una struttura residenziale psichiatrica per un trattamento riabilitativo con un supporto assistenziale variabile. L'inserimento in una struttura residenziale avviene esclusivamente a cura del Centro di Salute Mentale, tramite una procedura di consenso professionale per una buona pratica clinica, relativa a criteri di appropriatezza e condizioni che suggeriscono il ricorso ad un trattamento in regime residenziale, come processo attivo e non autorizzazione "passiva". Questa procedura si svolge, ove possibile, mediante la sottoscrizione di un "accordo/impegno di cura" tra DSM e utente, con la partecipazione delle famiglie e il possibile coinvolgimento della rete sociale, al fine di consentire la volontarietà e l'adesione del paziente al trattamento.

${ }^{80}$ Foglietta, F., “La integrazione socio-sanitaria nei Led”, in Sanità Pubblica e Privata, 2006, 3, pp. 8 y ss.

${ }^{81}$ I Piani di trattamento individuali (PTI) dei pazienti definiti dai Centri di Salute Mentale devono riferirsi a specifici criteri diagnostici, considerando altresì la gravità e complessità del quadro clinico, la compromissione del funzionamento personale e sociale del paziente, da stabilire sulla base di strumenti di valutazione standardizzati (ad es., HoNOS, BPRS, FPS, VADO), le risorse o potenzialità riabilitative, le resistenze al cambiamento, la stabilità clinica. Le
} 
Anche in questa prospettiva, nel contesto della salute mentale, si ritiene auspicabile, con riferimento ai LEA, il superamento del modello assistenziale per i disturbi mentali basato sul concetto di prestazioni, ripensando invece ad un approccio che individui una sequenza di processi o di livelli all'interno di ogni singolo progetto terapeutico e riabilitativo.

Attraverso i LEA è necessario che venga garantita una risposta personalizzata, affinchè ciascun utente possa addizionare al suo capitale sociale un budget di salute misurato sulla sua singolarità, sulla sua diversità, sul suo bisogno. In particolare, specie nella definizione di percorsi di cura ed assistenza di lungo periodo, si dovrà tener conto dell'intero percorso esistenziale della persona sofferente (intesa come globalità del processo di riproduzione sociale), rendendo accessibili sia interventi di tipo integrato (Servizi di salute mentale, Distretti sanitari e Servizi sociali) sia le risorse messe a disposizione dalla comunità (volontariato, cooperative, associazioni), che possano interagire in modo virtuoso con il contesto familiare, ambientale e sociale della persona. $^{82}$

In questi termini il concetto di livello essenziale viene inteso come 'percorsi di presa in carico e di cura esigibili', e non come singole prestazioni, tenuto conto della particolare complessità, multifattorialità e necessità di trattamenti integrati per i disturbi psichiatrici maggiori, ma anche in relazione ai fattori di rischio biopsicosociale e agli interventi di riabilitazione ed inclusione sociale. ${ }^{83}$

I servizi devono essere centrati sulla persona, che deve essere coinvolta in tutte le fasi del processo diagnostico, terapeutico e riabilitativo, sulla base di un approccio centrato sul desiderio di guarigione, sulle potenzialità di ripresa e di integrazione sociale del paziente, in cui gli standards riferiti alle strutture e alle offerte di prestazioni assumono solo un ruolo marginale.

Vista in questi termini, l'assistenza psichiatrica al malato mentale è sicuramente un problema di salute mentale della collettività, non l'unico e forse neppure il prevalente, laddove ai servizi si affaccia infatti una utenza nuova, ugualmente sofferente anche se meno disabile e disfunzionale,

risultanze di tali valutazioni orientano, da un lato, sull'intensità del trattamento riabilitativo e, dall'altro, sul livello assistenziale e tutelare da prevedere. L'obiettivo è quello di individuare una risposta appropriata ai bisogni specifici del paziente stabilendo una correlazione a due livelli, quello di intervento terapeutico riabilitativo e assistenziale necessario, in funzione di una prospettiva che guardi all'integralità esistenziale della persona.

${ }^{82}$ In questo senso, anche nel quadro dei LEA, si debbono definire standards che garantiscano prima di tutto l'accesso alle cure nel momento della crisi acuta o dell'urgenza psichiatrica, senza per questo ricorrere necessariamente al T.s.o., laddove possano intervenire servizi diffusi sul territorio a bassa soglia, capaci di muoversi verso la persona nel suo contesto domiciliare.

${ }^{83}$ Si tratterà di operare una sorta di cambiamento di paradigma: non più e non solo standard riferiti a strutture e offerte (posti letto, rette, drg), ma un approccio centrato sulle persone e sui processi che costruiscono prestando attenzione alle potenzialità di ripresa e al desiderio di guarigione che ognuno porta con sè. 
e perciò stesso più esigente e capace di tutelare i propri diritti alla salute: sono indicativi di tale fenomeno l'aumento del disagio giovanile, delle depressioni e dei disturbi ansiosi nella infanzia, nella adolescenza, nella giovane età adulta, dei disturbi del comportamento alimentare, dei disturbi di personalità, di tutte le suddette condizioni complicate dall'uso di sostanze, dei disturbi propri dell'anziano; si segnalano inoltre per le loro particolari espressività i disturbi mentali nei migranti di prima e seconda generazione. ${ }^{84}$ Sono capitoli ancora da scrivere nell'approccio alla salute mentale di comunità.

In tale contesto sembra comunque rilevante segnalare il problema della salute mentale in età geriatrica che si porrà in prospettiva come un questione preponderante: l'aumento dell'aspettativa di vita ha favorito l'estendersi della morbilità psichiatrica a una fascia di popolazione sempre più ampia, così, se è vero ad esempio che entro il prossimo quinquennio il numero di anziani ultrasessantacinquenni costituirà il 15-20\% della popolazione generale, almeno il 70\% di essi presenterà problemi psichiatrici o psicologici.

Proprio la consapevolezza che la frequenza delle demenze (in particolare l'Alzheimer) rischia di assumere, nell'immediato futuro, le caratteristiche di un fenomeno epidemico, soprattutto per la stretta relazione con il processo di invecchiamento della popolazione, ha convogliato su questa problematica una crescente attenzione dei responsabili della programmazione in sanità pubblica a livello nazionale nazionale. ${ }^{85}$

\footnotetext{
${ }^{84}$ In particolare, con riferimento specifico ai rifugiati e richiedenti asilo, il decreto del Ministero dell'Interno del 5 agosto 2010 ha previsto la possibilità di realizzare interventi di accoglienza specificatamente dedicati alla presa in carico di persone con disagio mentale, di carattere psicologico e psichiatrico. "Servizio centrale del Sistema di protezione per richiedenti asilo e rifugiati", Manuale operativo per l'attivazione e la gestione di servizi di accoglienza e di integrazione per richiedenti e titolari di protezione internazionale, Roma, 2011, pp. 64 y ss.

${ }^{85}$ Negli ultimi anni un riconoscimento importante alla centralità della tematica della demenza è venuto dalla sua inclusione, per la prima volta, nella pianificazione definita dal Piano Nazionale della Prevenzione (PNP) 2010-2012, in cui i punti di attenzione per le demenze sono stati identificati nel governo clinico complessivo, nella promozione delle migliori pratiche basate su evidenze, nella individuazione dei percorsi assistenziali integrati. Una serie di "azioni di supporto centrali" alla programmazione regionale dovrebbero andare, come è nella tradizione della programmazione del PNP, ad integrare le specifiche attività locali. Cruciali sono, infatti, i Piani Regionali Prevenzione (PRP) che discendono in modo diretto dal quadro di riferimento nazionale, ma sono calibrati sulle esigenze e sulle priorità locali. Per affiancare questa progettualità ad ampio spettro, il Ministero ha previsto lo stanziamento di fondi vincolati per I'Assistenza ai pazienti affetti da Demenza, all'interno Obiettivi di Piano per le Regioni, sia per l'anno 2010 (Accordo Stato-Regioni dell'8/7/2010) che per il 2011 (Accordo Stato-Regioni del 20/4/2011). Si tratta di obiettivi strategici e prioritari sui quali si fa convergere una parte vincolata del Fondo sanitario nazionale. Si veda A. Bianchetti, A. Y Trabucchi, M., Alzheimer, Bologna, Il Mulino, 2010; Di Fiandra, T., "Le politiche per le demenze in Italia e in Europa", in AA. VV., Home Care. Le cure domiciliari nella medicina della complessità, Atti del IV Congresso europeo, Milano, 2012, pp. 66 y ss.
} 
Per affrontare la questione, al di là di quanto già previsto a livello nazionale, in molte regioni sono stati avviati servizi residenziali e domiciliari, integrativi dei LEA, finalizzati all'offerta di prestazioni psicogeriatriche di tipo multidisciplinare che si strutturano attraverso l'azione di competenze diverse e complementari, con compiti di identificazione della patologia, di verifica dell'ipotesi diagnostica, di consulenza e collegamento operativo e di progettazione di piani di intervento. $^{86}$

Quanto all'approccio ai nuovi bisogni che emergono nella società, si nota con favore la previsione dell'articolo 5 del d.Ig. 13 settembre 2012, núm. 158, che prevede l'inserimento nei LEA (il cui aggiornamento è in fase di approvazione) delle prestazioni di prevenzione, cura e riabilitazione della ludopatia. In attuazione del dettato normativo, il provvedimento include esplicitamente le persone affette da questo tipo di dipendenza tra coloro cui sono rivolti i servizi territoriali per le dipendenze (Sert, Centri diurni, ecc.) già attivi nelle Asl, e modifica la definizione del sotto-livello di assistenza, attualmente riportata nel dPCM del 2001 come "Attività riabilitativa sanitaria e sociosanitaria rivolta alle persone dipendenti da sostanze stupefacenti o psicotrope o da alcool", riformulandola come "Assistenza socio sanitaria alle persone con dipendenze patologiche o comportamenti di abuso di sostanze", innovando in conformità ai criteri delle scienze psichiatriche relativi alle moderne dipendenze e, al contempo, affermando in tal modo il principio per cui le persone affette da ludopatia hanno diritto ad accedere al Servizio sanitario per ricevere le prestazioni di cui hanno bisogno al pari dei soggetti con altre forme di dipendenze patologiche. $^{87}$

Per completare il quadro si può notare che, come per altri ambiti dell'assistenza sanitaria, anche per la salute mentale negli anni recenti sono stati ridefiniti i domini concettuali che sottendono alla continuità delle cure, da intendere non solo come continuità relazionale tra operatore e paziente ma anche e soprattutto come coordinamento tra setting assistenziali (ospedale-territorio), operatori e prestazioni. Fa parte del concetto di continuità assistenziale anche la continuità informativa (informational continuity), che consiste nella registrazione e trasmissione delle informazioni che riguardano i pazienti come presupposto per un corretto coordinamento e integrazione dei servizi offerti da parte di diversi operatori in diversi setting assistenziali. Sicchè, anche di queste innovazioni si dovrà tener conto nella definizione dei livelli essenziali, nella misura in cui solo strutturando i servizi e le prestazioni attraverso una rete di

\footnotetext{
${ }^{86}$ Per riferimenti A. Golini, A. Rosina (a cura di), Il secolo degli anziani, Bologna, Il Mulino, 2013, pp. 21 y ss.; Micheli, G. A. (a cura di), La questione anziana. Ridisegnare le coordinate di una società che invecchia, Milano, Franco Angeli, 2004, pp. 22 y ss.

${ }^{87}$ Sul punto Bianchetti, R., "I giocatori compulsivi", in Cendon, P. y Rossi, S. (a cura di), I nuovi danni alla persona. I soggetti deboli, Roma, Aracne, t. II, pp. 859 y ss.
} 
percorsi integrati si potrà permettere al paziente di progredire in maniera evolutiva a seconda dei propri bisogni di assistenza.

\section{SCIENZA INCERTA E MENTAL HEALTH POLICY}

Nell'approccio alle tematiche della salute mentale è necessario evitare di cadere nell'equivoco dell' "ubriaco sotto il lampione", ovvero dell'uomo che ha perso le chiavi di casa e continua a cercarle sotto il lampione, non perché abbia motivo di pensare che le troverà in quel luogo, ma perché quello è l'unico posto illuminato. Tuttavia la luce del lampione non è eterna e ad un certo punto si dovrà cercare altrove. Fuor di metafora si può osservare che questa centralità del richiamo al sapere scientifico e tecnico dipende dal tentativo di ridurre problemi politico-pratici a questioni che si ritiene possano essere risolte sulla base di criteri asettici, che non presuppongono scelte etiche o un approccio integrato. Nel campo della salute mentale, specie nelle scelte di carattere normativo, si danno per presupposte troppe certezze che in realtà latitano: al disturbo mentale ${ }^{88}$ è riservato infatti uno statuto particolare, una condizione di instabilità che rende la sofferenza psichica uno spazio di ricerca e di confronto polemico, anziché un luogo di incontro irenico.

È proprio sul versante dei sicuri ancoraggi scientifici che la questione del disturbo mentale presenta i più rilevanti aspetti di problematicità, in un contesto in cui la dottrina parla, pressoché unanimemente, di "crisi della psichiatria", di "una crisi di identità... da alcuni anni attraversata" dalla scienza psichiatrica, risultando "la classificazione dei disturbi psichici quanto mai ardua e relativa, non solo per la mancanza di una terminologia generalmente accettata, ma per i profondi contrasti esistenti nella letteratura psichiatrica". ${ }^{89}$ La scienza psichiatrica propone, difatti, come è noto, paradigmi e modelli scientifici diversi e tra loro conflittuali: dal paradigma organicistico, per cui le infermità mentali sono vere e proprie malattie del cervello o del sistema nervoso, aventi, per ciò, un substrato organico o biologico, a quello psicanalitico per cui i disturbi mentali

\footnotetext{
${ }^{88}$ In tal senso si riprende l'analisi di Dell'Acqua per cui è più corretto utilizzare la nozione di disturbo mentale e non malattia mentale, volendo sottolineare l'impossibilità di omologarlo al paradigma medico. Dell'Acqua, P., Persone, malattia mentale e guarigione, in L. Lenti, L. et al. (a cura di), I diritti in medicina, Trattato di biodiritto, diretto da S. Rodotà e P. Zatti, Milano, Giuffrè, 2011, 783, núm. 1.

${ }^{89}$ In questo senso Cass. pen., sez. un., 8 marzo 2005, n. 9136, in Cass. pen., 2005, 3, p. 120. In dottrina McHugh, P. R. y Slavney, P. R., The perspectives of psychiatry, Baltimore, John Hopkins University Press, 1986; Poli, E. y Cioni, P., "Modelli di malattia in psichiatria", in Pancheri, P. y. Cassano, G. B. (a cura di), Trattato italiano di psichiatria, Milano, Masson, 1999. In controtendenza Rossi, R., "Psichiatria o psichiatra che cambia? Vicende evolutive dello psichiatra", Italian Journal of Psychopathology, 2005, 11, 4, p. 412. Secondo cui "forse non c'è bisogno di inseguire statuti ineccepibili che ci diano sicurezza, e non c'è bisogno di cercarli e di adattarsi: I'attrezzatura dello psichiatra è una attrezzatura mentale, di per sé specifica e non comune, che fa sua la sofferenza, la tollera perché la elabora e la racconta, ricostruisce la trama di una vita interiore fratturata, fornendo idee, narrative, teorie, conoscenze biologiche, sociologiche, letterarie. Basta e avanza...".
} 
rappresentano disarmonie dell'apparato psichico, o quello sociologico, per il quale la malattia mentale è disturbo psicologico avente origine sociale, non più attribuibile ad una causa individuale di natura organica o psicologica, ma a relazioni inadeguate nell'ambiente in cui il soggetto vive. Nell'ultimo decennio hanno trovano infine nuovo spazio gli orientamenti ispirati ad una prevalenza del dato medico (psichiatria c.d. biologica legata allo studio delle neuroscienze), valorizzanti l'eziologia biologica della malattia mentale.

Né può dirsi risolutivo il modello enucleato dal Manuale Diagnostico e statistico dei disturbi mentali, il DSM-V, messo a punto dall'American Psychiatric Association nel 2012, che declina con una nomenclatura nosografica che richiama sindromi e non malattie - i principali disturbi mentali in diciassette classi diagnostiche, in quanto il c.d. "approccio ateorico", fondato utilizzando dati descrittivi o "obiettivi", se viene utilizzato nella clinica, dimenticando le complesse variabili del rapporto interpersonale, snatura la funzione della psichiatria e rischia di offrire l'immagine di un paziente che esiste solo sul tavolo autoptico, ma non nella realtà clinica. ${ }^{90}$

Si profila quindi, anche all'attenzione del nomoteta, un contesto culturale e scientifico caratterizzato da complessi e molteplici modelli teorici e dal confronto tra diversi orientamenti di epistemologia psichiatrica, il che inevitabilmente comporta ricadute sulla definizione di alternative opzioni legislative, regolamentari e di riorganizzazione dei servizi (dal confronto sull'utilità del concetto di capacità di intendere e volere ${ }^{91}$ e di imputabilità, ${ }^{92}$ alle questioni connesse all'espressione del consenso alle terapie, ${ }^{93}$ sino agli strumenti adottati dalla legislazione in materia di tutela della salute mentale), ponendo il legislatore di fronte alla sfida di delineare

\footnotetext{
90 Demazeux, S., Qu'est-ce que le DSM? Genèse et transformations de la bible amèricaine de la psychiatrie, Paris, Parution, 2013, pp. 25 y ss.; Wakefield, J. C., “DSM-5: An Overview of Changes and Controversies”, Clinical Social Work Journal, 2013, 41, 2, p. 139 ss.; Wakefield, J. B y First, M. B., “Classifying the distinction between disorder and nondisorder: confronting the overdisagnosis (false positive) problem in DSM-IV", in Philips, K. A., First, M. B., y Pincus, H. A. (eds), Advancing DSM: Dilemmas in psychiatric diagnosis, Washington, Arligston, American Psychiatric Association, 2003, pp. 126 y ss.

${ }^{91}$ Zatti, P., "Oltre la capacità di intendere e volere”, in Ferrando, G. y Visintini, G. (a cura di), Follia e diritto, Torino, Bollati Boringhieri, 2003, pp. 49 y ss.; Visintini, G., “La nuova disciplina sui trattamenti sanitari obbligatori per malattie mentali. Riflessi sulla nozione di incapacità di intendere e volere", Politica del diritto, 1984, pp. 817 y ss.

${ }^{92}$ Visintini, G., "La crisi della nozione di imputabilità nel diritto civile”, in Ferrando, G. y Visintini, G. (a cura di), op. cit., pp. 189 y ss.; Monteverde, L., La nozione di imputabilità penale, cit., pp. 201 y ss.

${ }^{93}$ Venchiarutti, A., “Obbligo e consenso nel trattamento della sofferenza psichica”, in Lenti, L. et al., (a cura di), I diritti in medicina, Trattato di biodiritto, cit., p. 817 ss.
} 
categorie e concetti, dotati di propria autonomia, ma in grado di dialogare con il complesso sapere delle scienze psichiatriche. ${ }^{94}$

In questa prospettiva pare utile la valorizzazione di quegli orientamenti che affermano un "modello integrato" della malattia mentale, in grado di spiegare il disturbo psichico sulla base di diverse ipotesi esplicative della sua natura e della sua origine: trattasi, in sostanza, di "una visione integrata, che tenga conto di tutte le variabili, biologiche, psicologiche, sociali, relazionali, che entrano in gioco nel determinismo della malattia", in tal guisa superandosi la visione eziologica monocausale della malattia mentale, e pervenendo ad una concezione "multifattoriale integrata" in un'ottica olistica contraddistinta dalla complementarità e dalla circolarità. ${ }^{95}$

A questa concezione multifattoriale del disturbo mentale si accompagna un approccio complesso, di comunità, dei servizi dedicati alla prevenzione e cura in tema di salute mentale, visioni che sono state fatte proprie dal legislatore - a partire dalla I. 180/1978 - sia nella valutazione degli strumenti e delle terapie di approccio al disturbo che nell'organizzazione dei servizi, che si plasmano nelle forme di intervento sui bisogni dell'utente. Proprio per tale motivo riteniamo che la legislazione psichiatrica possa e debba essere implementata (attraverso i Progetti Obiettivo, le Linee di Azioni nazionali per la salute mentale, i Piani regionali), ma non certo riformata, nella misura in cui le innovazioni che essa ha introdotto nel sistema della salute mentale sono state di per sé in grado di incidere nella carne del sistema e nell'esistenza degli individui, suscitando autonomamente dinamiche di conflitto, di adattamento e di trasformazione. ${ }^{96}$

Questo rapporto tra modelli scientifici e modelli normativi corrisponde peraltro all'indirizzo definito dalla consolidata giurisprudenza costituzionale secondo cui "salvo che entrino in gioco altri diritti o doveri costituzionali, non è, di norma, il legislatore a poter stabilire direttamente e specificamente quali siano le pratiche terapeutiche ammesse, con quali limiti e quali condizioni... la regola di fondo in questa materia è costituita dalla autonomia e dalla responsabilità del medico... sempre con il consenso del paziente". (Corte Costituzione, núm. 282/2002). E ancora: "Scelte legislative dirette a limitare o vietare il ricorso a determinate terapie... non sono

\footnotetext{
${ }^{94}$ Carozza, P., La psichiatria di comunità tra scienza e soggettività: linee di pratica clinica nei servizi di salute mentale, Milano, Franco Angeli, 2010.

${ }^{95}$ Schiavone, M., "Psichiatria e salute mentale", in Comitato Nazionale per la Bioetica, I/ Comitato Nazionale per la Bioetica: 1990-2005. Quindici anni di impegno, Presidenza del Consiglio dei Ministri, Roma, Dipartimento per l'Editoria, 2006, p. 495.

${ }^{96}$ Rossi, S., "Sussurri e grida. Riflessioni su follia e libertà nell'ennesimo testo di riforma della legge Basaglia”, Politica del diritto, 2012, 2-3, p. 470.
} 
ammissibili ove nascano da pure valutazioni di discrezionalità politica", senza connessione con conoscenze scientifiche ed evidenze sperimentali (Corte Costituzione, núm. 338/2003).

Entro questo schema il rapporto tra diritto e scienza (psichiatrica) vive infatti su binari che non si muovono in una sola direzione, ma che si intrecciano, si scambiano i ruoli, creando uno schema di concorrenza nella produzione di regole e contenuti in cui, da un lato, la conoscenza del contesto normativo è necessario presupposto alla comprensione della scienza e delle pratiche che l'inverano, e, dall'altro, la scienza è fonte di numerosi cambiamenti normativi. Così se, in alcuni casi, la scelta tra diverse soluzioni (di disciplina o di organizzazione) normativa finisce col dipendere dal modo in cui il sapere scientifico tematizza e configura un aspetto controverso, al contempo, tuttavia, l'intervento del diritto non si configura in forma di semplice ratifica, specie quando la traduzione giuridica dell'acquisizione scientifica svolge una funzione di validazione, di adattamento entro un contesto di regole consolidate. ${ }^{97}$

In questa prospettiva, attualmente, le due maggiori sfide con le quali gli operatori della salute mentale e per il legislatore che deve recepirne finalità e obiettivi si misurano per aumentare la qualità dei servizi psichiatrici sono: a) ridisegnare i servizi sui principi del recovery, $b$ ) implementare servizi e pratiche sostenuti dall'evidenza scientifica.

a) Il recoverysi realizza quando le persone con sofferenza mentale scoprono o riscoprono le loro abilità e le loro risorse per perseguire obiettivi personali e sviluppare un senso di sè che permette loro di crescere, superando o marginalizzando la loro malattia mentale. ${ }^{98}$

Il recovery è quindi un processo soggettivo e oggettivo che deve essere riguardato all'interno di un continuum e non come nella dicotomia guarito/non guarito. Indirizzare l'azione dei servizi psichiatrici ospedalieri e di territorio, delle agenzie informali (volontariato) e della famiglia verso la prospettiva del recovery significa garantire al paziente attraverso l'azione terapeutica e il supporto sociale: a) aree indipendenti di funzionamento (emotive, cognitive, sociali, lavorative e spirituali); b) adattamenti psicosociali alla disabilità; c) raggiungimento di uno stato soggettivo di benessere; d) miglioramenti nel funzionamento di ruolo e nell'integrazione nella comunità.

In tale contesto si inseriscono le strategie utili ad attivare le migliori modalità per favorire la ricerca e sperimentazione di forme di concertazione locale e di partecipazione funzionali al conseguimento di alcuni principi di riferimento secondo cui:

— non si può dare nessun territorio senza comunità che lo rappresenti; -

\footnotetext{
97 D'Aloia, A., "Biodiritto oltre lo Stato", in Pomarici, U. (a cura di), Atlante di filosofia del diritto, Torino, Giappichelli, t. II, 2013, pp. 38 y ss.

${ }^{98}$ Mueser, K. T. et alt., “Illness Management and Recovery: A Review of the Research”, Psychiatric Service, 2002, 53, 10, pp. 1272 y ss.
} 
— è solo attraverso relazioni organizzate che si assicura la partecipazione;

— senza relazioni organizzate non può essere dato nessun sistema informativo e viceversa;

— si devono definire le questioni che rientrano in un negoziato sociale;

- ripensare il modo di concepire la partecipazione significa ripensare il modo di essere delle istituzione dei servizi a partire dall'azienda.

I dipartimenti di salute mentale -in linea e in ottemperanza alla filosofia sottesa alla riforma- devono ritagliarsi un ruolo attivo nei confronti dell'interlocutore sociale per riformulare una negoziazione sociale sull'oggetto del loro lavoro e per definire quali sono le problematiche emergenti nei singoli territori. Tutto ciò al fine di rispondere all'esigenza di intervenire in modo tempestivo sulle persone con disagio mentale per evitare il rischio di atrofizzare le loro capacità e per reinserirle in un percorso di riappropriazione delle opportunità di cittadinanza, facendo leva sul loro empowerment e sulla collaborazione dei diversi attori e servizi di prossimità della comunità.

Si è sviluppata, per superare il limite dei servizi formali, la creazione di servizi integrati nella comunità, collocati, cioè, nel luogo dove insorgono i bisogni e dove si alimenta l'intreccio naturale delle risorse umane. Si è costituita e specializzata tipologicamente la 'rete formale di supporto comunitario', a composizione mista pubblico-privato. A sviluppo di ciò, si individua nella cooperazione sociale il soggetto associativo potenzialmente più adatto ad operare sul terreno della promozione dei diritti di solidarietà e su quello della erogazione delle prestazioni attraverso le quali tali diritti si concretizzano.

Al contempo, in ambito sociale, il principio di sussidiarietà orizzontale legittima i soggetti della comunità territoriale anche non formalmente costituiti a farsi carico a pieno titolo delle aree problematiche e di sofferenza personale e sociale. Si tratta di favorire una cultura che riconosca la caratterizzazione della rete sociale come "comunità terapeutica riabilitativa allargata" (attraverso la nascita di gruppi di self-help, la creazione di reali momenti partecipativi, di occasioni di assunzioni di responsabilità e potere decisionale degli utenti), in grado di operare con caratteristiche relazionali, oltre che formali, strutturate e regolative.

b) Per superare la crisi della psichiatria, è necessario rifarsi ad una pratica clinica basata su prove di efficacia riferentesi all'utilizzo, all'interno del processo decisionale terapeutico, delle migliori prove di evidenza scientifica (Ebp/Ebn). ${ }^{99}$

\footnotetext{
${ }^{99}$ Il concetto di Ebn ha generato un notevole interesse per la stretta connessione con i problemi largamente riportati associati all'adozione e utilizzo nella pratica clinica dei risultati delle ricerche condotte in ambito sanitario. La medicina basata su prove di efficacia viene definita come il "conscienzioso e giudizioso utilizzo della migliore evidenza disponibile per prendere decisioni riguardo l'assistenza dei singoli pazienti. Comporta il combinare le evidenze sperimentali migliori disponibili con l'esperienza clinica e i valori dei pazienti”. Sackett et al., "Evidence-based medicine:
} 
L'attuale sviluppo della ricerca scientifica per avanzare nelle conoscenze dei fenomeni complessi pone, inoltre, la necessità di valutare gli esiti di efficacia dovuti all'applicazione delle nuove teorie e tecnologie. ${ }^{100}$

Le esperienze realizzate dai servizi della salute mentale, previsti dalla riforma del 1978, vanno studiate e valutate, dunque, in relazione alla introduzione dei nuovi saperi e ai diversi modelli di applicazione: ${ }^{101}$ l'approccio alla salute mentale mette infatti insieme competenze e saperi anche assai diversi fra loro, allargando il suo campo di interessi dagli aspetti medico-biologici, a quelli psicologici e psicosociali. La misura del benessere o della riduzione del disagio del paziente psichiatrico emerge dall'azione combinata di pratiche professionali che focalizzano il loro intervento sull'azione di agenti chimici, ambienti e comportamenti sociali, moduli organizzativi e gestionali.

what it is and what is isn't", British Medical Journal, 1996, 312, p. 71. Inoltre gli autori affermano che essa si struttura come un processo di integrazione fra l'abilità clinica del singolo operatore e la migliore evidenza clinica esterna che deriva da ricerca sistematica. Una delle conseguenze di questi sviluppi è la compilazione degli elenchi dei cosiddetti Empirically Supported Treatments (Est), cioè dei trattamenti psicoterapeutici supportati empiricamente o evidencebased (basati sulle evidenze), che sarebbero le uniche terapie che funzionano mentre tutte le altre non dovrebbero più essere praticate (non pare secondario dire che tra queste terapie dichiarate 'inutili' vi sarebbero quasi tutte le terapie psicoanalitiche). Ciò in quanto la psichiatria evidence based si fonda su una valutazione critica degli interventi diagnostici, farmacologici e psicoterapeutici alla luce dell'epidemiologia clinica e della revisione sistematica della letteratura disponibile.

${ }^{100}$ Sebbene alcune delle più grandi modificazioni intervenute in questi decenni nell'organizzazione dei servizi di salute mentale non siano ascrivibili primariamente alla spinta o ai risultati della ricerca, ma siano state portate avanti sull'onda di grandi spinte ideali e culturali, le conoscenze acquisite in questi ultimi tre decenni sia sulle neuroscienze e sui meccanismi eziopatogenetici dei disturbi mentali gravi, che sull'efficacia dei trattamenti farmacologici e psicosociali, hanno profondamente modificato l'orizzonte concettuale ed operativo dell'intero campo della salute mentale. Le opzioni oggi presenti, che spesso si confrontano tra loro, sono in massima parte eticamente e culturalmente accettabili, e quindi una scelta appropriata tra diverse opzioni organizzative e di trattamento dovrebbe in larga misura fare riferimento a dati quanti- e qualitativi ottenuti in studi condotti con metodologie appropriate: tale assunto è alla base della crescita impetuosa della evidence-based medicine in psichiatria.

${ }^{101}$ Per quanto riguarda le malattie mentali, in questi anni vi è notoriamente un fervore nella ricerca biologica (cd. neuroscienze), in particolare articolandosi in: a) ricerche sui neutrotrasmettitori, ricettori e psicofarmaci, che tendono a convalidare la concezione delle psicosi endogene come malattie a patogenesi sui generis, cioè correlata a specifiche, anche se tuttora non completamente chiarite, disfunzioni recettoriali e/o neurotrasmettitoriali; b) individuazione di marker biologici, ovvero variabili biologiche correlate a qualche situazione clinica, di cui possono fungere da indicatori attraverso cui una prima validazione di sindromi cliniche finora definite solo sintomatologicamente; c) interazione geni/ambientale e plasticità del sistema nervoso, ricerche che si fondano sulla proposta di modelli genetici più complessi di quelli tradizionali, per cui lo studio dell'interazione tra apprendimento e rete neuronale (plasticità neuronale) è in grado di prospettare un superamento della semplicistica dicotomia eredità/ambiente. L'idea che la malattia mentale sia espressione di un programma genetico non è affatto incompatibile con il peso di interferenze ambientali. 


\section{CONCLUSIONI MINIME: LA SALUTE MENTALE TRA LEGISLAZIONE DI DETTAGLIO E LINEE GUIDA}

Abbiamo già illustrato come, solo nella seconda metà degli anni '90, sia stato possibile affrontare in maniera organica i problemi e le carenze normative in materia di salute mentale, cercando di dare risposte concrete attraverso i Progetti Obiettivo nazionali 1994-1996 e 1998-2000, che hanno finalmente offerto agli amministratori, operatori e agli altri soggetti interessati l'opportunità di un confronto concreto sui modelli organizzativi, sulle direttive e le finalità dei servizi psichiatrici ospedalieri e territoriali. Entrambi i Progetti Obiettivo furono accompagnati da un momento di particolare vitalità dei servizi, e di sforzi per la realizzazione di azioni «a ventaglio» volte al compimento di passi avanti nelle molteplici direzioni che venivano individuate.

Dal 2000 le regioni hanno dimostrato un forte attivismo affrontando il discorso dell'organizzazione dell'assistenza psichiatrica attraverso la legislazione, la regolamentazione e programmazione regionale. ${ }^{102}$

È bene dire che le normative nazionali non hanno trovato applicazione uniforme a livello delle singole regioni, portando a differenze, talvolta sostanziali, nelle possibilità di cura del cittadino in relazione al luogo di vita. Laddove le normative non sono state applicate in modo completo, 0 non sono state affatto applicate, per disimpegno politico o incapacità amministrativa, sono conseguite carenze negli interventi sociosanitari per la psichiatria a livello regionale e locale.

Solo dal 2006 si è ritornati ad esaminare le tematiche connesse alla salute mentale in tutte le fasi della vita ed i sistemi di psichiatria di comunità, attraverso un complesso di iniziative ministeriali ed interregionali che si sono mosse nella logica della continuità e delle innovazione ed hanno portato all'emanazione di atti d'intesa della Conferenza Stato-Regioni, raccomandazioni della Conferenza delle Regioni ${ }^{103}$ e Dpcm di particolare importanza: le Linee di indirizzo nazionale

\footnotetext{
${ }^{102}$ In questo senso si vedano, a titolo d'esempio, le leggi regionali in materia sanitaria (L.r. Emilia Romagna 2/2003 e 29/2004; Lombardia 31/1997), i Piani regionali per la salute mentale (PRSM 2004 della Lombardia; Piano Attuativo Salute Mentale 2009 dell'Emilia Romagna; Progetto Obiettivo Salute Mentale 2010 del Veneto). Nella programmazione di tutte le Regioni vi sono indicazioni sul ruolo dei Dipartimenti di salute mentale nel collegamento con i diversi soggetti presenti sul territorio: così principali interlocutori istituzionali del territorio sono i DSM, DSS, i Piani di Zona, mentre in alcune regioni hanno un ruolo di coordinamento i DSM (es. Lazio) o i DSS (es. Veneto, Emilia Romagna). In alcune regioni sono stati istituiti organismi ad hoc per rendere effettivo il coordinamento (es. Trentino: Comitati di Coordinamento locali per la psichiatria; Lombardia: Organismi di Coordinamento per la Salute Mentale; Liguria: Comitato dei Distretti Sociosanitari).

${ }^{103}$ Sono da segnalare le 'Raccomandazioni in merito all'applicazione di accertamenti e trattamenti sanitari obbligatori per malattia mentale (artt. 33-34-35 Legge 23 dicembre 1978, n. 833) approvate dalla Conferenza delle Regioni e Province autonome in data 29 aprile 2009; 'Contenzione psichica in psichiatria: una strategia possibile di prevenzione' approvata dalla Conferenza delle Regioni e Province autonome in data 29 luglio 2010; 'Le strutture residenziali psichiatriche approvata dalla Conferenza delle Regioni e Province autonome in data 13 giugno 2013.
} 
per la salute mentale 2008; il Dpcm 1 aprile 2008 sulla sanità penitenziaria e sul superamento degli Opg ${ }^{104}$; il Sistema informativo nazionale istituito con d.m. 15 ottobre 2010; il Piano nazionale autismo ed infine il Piano di Azioni nazionali per la salute mentale del 2013.

Il sistema dei servizi di salute mentale e le norme che lo disciplinano si mostra come "un insieme di nodi interconnessi", ${ }^{105}$ una rete che si presta a rappresentare la forma organizzativa dei legami tra istituzioni, territorio e cittadinanza (intesa anche come insieme degli utenti).

Come si è cercato di mostrare, le differenze tra i sistemi regionali — pur profonde- ${ }^{106}$ vengono ad essere mitigate dall'adozione di una forma organizzativa che privilegia i «coordinamenti orizzontali» e un orientamento processuale, attraverso il quale si compie appunto il passaggio dalla gerarchia alla rete.

In questa prospettiva che caratterizza di per sé i servizi territoriali, vi si può riconoscere una grande forza normativa che si invera in un'intensa opera di smontaggio dell'architettura verticale delle istituzioni -almeno di quelle ante legge Basaglia - e al tempo stesso crea una mobilitazione di una grande quantità di energie pratiche e morali di trasformazione attorno al principio dell'auto-organizzazione, e alla riorganizzazione «orizzontale», in cui sono riconoscibili processi istituenti.

Seppure non sia facile praticare e insieme governare queste dimensioni, tuttavia è la natura sottile della legge 180, la sua finalità di decostruzione dell'istituzione, a renderla sistema normativo prospettico, che da vita ad un "diritto omeostatico", ${ }^{107}$ capace di autoadattamento, di seguire il costante mutamento determinato dalla scienza e dalle relazioni sociali.

Per organizzare un efficace politica di salute mentale, tale da fornire risposte ai bisogni vecchi e nuovi che il contesto sociale propone e che, prevedibilmente, si proporranno nel prossimo futuro, le regioni, gli enti locali e le forze sociali devono essere in grado di pianificare azioni e prassi da esercitare in modo coordinato e coerente facendo interagire il sistema di comunità con

\footnotetext{
${ }^{104}$ Normativa che ha trovato realizzazione, almeno formale, con la I. 17 febbraio 2012, núm. 9 su cui A. Pugiotto, “L'ergastolo nascosto (e altri orrori) dietro i muri degli ospedali psichiatrici giudiziarl", Quaderni costituzionali, 2013, 2, pp. 343 y ss.

${ }^{105}$ Castells, M., The Rise of the Network Society, Oxford, Blackwell,1996, p. 232.

${ }^{106}$ La differenziazione nell'organizzazione della sanità che si è realizzata tra le regioni italiane merita di essere tenuta sotto controllo, in quanto potrebbe essere foriera di forme di produzione e riproduzione delle disuguaglianze di salute alternative ai tradizionali cleavege di stratificazione sociale. Esemplificativa a questo riguardo è l'implementazione dei welfare regionali (il processo di decentramento delle responsabilità) a cui si accompagnano razionamenti delle prestazioni sanitarie e svantaggi per i soggetti più deboli.

107 Rodotà, R., “Diritto, scienza, tecnologia: modelli e scelte di regolamentazione”, in Comandè, G. y Ponzanelli, G. (a cura di), Scienza e diritto nel prisma del diritto comparato, Torino, Giappichelli, 2004, p. 409.
} 
il sistema di cura. È attraverso questa interazione che è possibile costruire uno dei pilastri del nuovo welfare di comunità basato sui concetti di personalizzazione ed integrazione. 